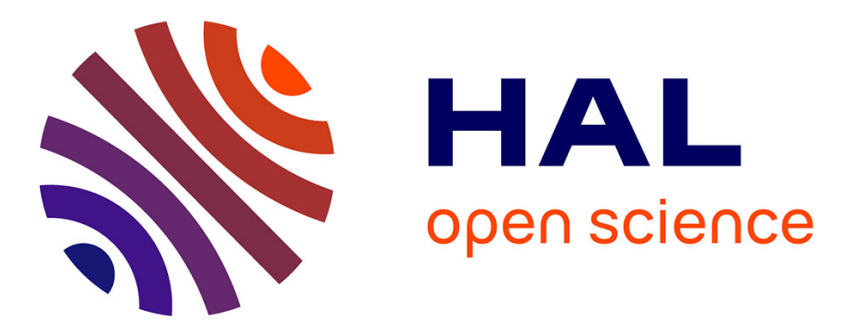

\title{
Externalities, consumption constraints and regular economies
}

\author{
Jean-Marc Bonnisseau, Elena L. del Mercato
}

\section{To cite this version:}

Jean-Marc Bonnisseau, Elena L. del Mercato. Externalities, consumption constraints and regular economies. 2008. halshs-00257731v2

\section{HAL Id: halshs-00257731 \\ https://shs.hal.science/halshs-00257731v2}

Submitted on 5 May 2009

HAL is a multi-disciplinary open access archive for the deposit and dissemination of scientific research documents, whether they are published or not. The documents may come from teaching and research institutions in France or abroad, or from public or private research centers.
L'archive ouverte pluridisciplinaire HAL, est destinée au dépôt et à la diffusion de documents scientifiques de niveau recherche, publiés ou non, émanant des établissements d'enseignement et de recherche français ou étrangers, des laboratoires publics ou privés. 


\section{Documents de Travail du Centre d'Economie de la Sorbonne}

C

E

$\mathrm{S}$

W

0

1

k

i

n

g

$\mathrm{P}$

a

p

e

1

$\mathrm{S}$

Version révisée

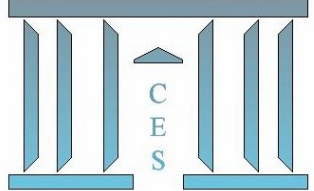
regular economies

\subsection{1}

Externalities, consumption constraints and

Jean-Marc BonnisseAU, Elena L. Del MERCATo 


\title{
Externalities, consumption constraints and regular economies $\star, \star \star$
}

\author{
Jean-Marc Bonnisseau and Elena L. del Mercato ${ }^{1}$ \\ First version: February 2, 2008. Revised version: April 7, 2009. \\ Accepted for publication on Economic Theory
}

\begin{abstract}
We consider a general model of pure exchange economies with consumption externalities. Households may have different consumption sets and each consumption set is described by a function called the possibility function. Utility and possibility functions depend on the consumptions of all households. Showing by means of an example that basic assumptions are not enough to guarantee generic regularity, we provide sufficient conditions for generic regularity in the space of endowments and possibility functions.
\end{abstract}

JEL classification: C62, D50, D62.

Key words: Externalities, consumption constraints, competitive equilibrium, regular economies.

\footnotetext{
* A la mémoire de Dave Cass.

${ }^{\star \star}$ We are deeply indebted to Andreu Mas-Colell for many precious and stimulating discussions, while Elena L. del Mercato was invited as visiting scholar to Department of Economics and Business, Universitat Pompeu Fabra. We wish to thank Paolo Siconolfi and Monique Florenzano for much encouragement and advice. Presented at the SAET Conference (2007), at the Conference on Economic Theory, University of Kansas (2007), and at the 4th Annual CARESS-Cowles Conference on General Equilibrium and Its Applications, University of Pennsylvania (2008), this version of the paper has also benefited from the comments of these audiences. We also thank two anonymous referees for their valuable remarks.

1 J.-M. Bonnisseau, Paris School of Economics, Université Paris 1 PanthéonSorbonne. Address: Centre d'Economie de la Sorbonne, 106-112 Boulevard de l'Hôpital, 75647 Paris Cedex 13, France. E-mail: Jean-Marc.Bonnisseau@univparis1.fr; E. L. del Mercato, Paris School of Economics, Université Paris 1 PanthéonSorbonne. Address: Centre d'Economie de la Sorbonne, 106-112 Boulevard de l'Hôpital, 75647 Paris Cedex 13, France. E-mail: Elena.delMercato@univ-paris1.fr
} 


\section{Introduction}

We consider a class of pure exchange economies with consumption externalities. Our goal is to provide sufficient conditions for the generic regularity.

In the presence of externalities, competitive equilibria are not necessarily Pareto optimal. It is therefore an open and important issue to study Pareto improving policies. Our regularity result is a first step in studying this issue, since the comparison of welfare at equilibrium before and after Pareto improving policies is possible only in a neighborhood of a regular economy. Indeed, in a regular economy, the number of equilibria is finite and the dependence of each equilibrium on the parameters describing the economy is locally continuous or differentiable, hence one can perform comparative static analysis. The analysis of Pareto improving policies in regular economies is justified only when regular economies are generic. In the presence of market failures, recent works utilize the generic set of regular economies as the starting point of several Pareto improving policies ${ }^{2}$.

There is a large and growing literature on general equilibrium models with externalities. Following Laffont and Laroque (1972), Laffont (1976, 1977, 1988) and Hammond (1998), we incorporate consumption externalities not only in preferences but also in consumption sets. That is, consumption sets depend on the consumption of the other agents. In the case of a network commodity like the internet or electricity, the available quantity and quality of the service depend on the global consumption. For example, congestion limits the physically possible individual consumptions. So, for a consumer who suffers negative external effects, the consumption set becomes "smaller" since less consumption opportunities are available to him. But, on the contrary, positive external effects may enlarge the individual consumption possibilities. For instance, positive effects due to the width of the network enables consumers to have a better service. Importantly, in both examples the external effects affect simply the individual consumption sets. They do not directly affect preferences.

So, because of the externalities, the consumption set is not necessarily the whole positive orthant of the commodity space. To model consumption sets possibly different from the set of positive consumptions, we follow Smale (1974), where each consumption set is described by an inequality on a differentiable function called the possibility function ${ }^{3}$. We simply assume that the

2 See, in different settings, Geanakoplos and Polemarchakis (1986, 2008), Cass and Citanna (1998), Citanna, Kajii and Villanacci (1998), Herings and Polemarchakis (2005), Citanna, Polemarchakis and Tirelli (2006).

3 In the same spirit are more recent works where portfolio sets are described in terms of linear or differentiable functions, Siconolfi $(1986,1988)$, Balasko, Cass and 
possibility functions depend on the consumption of the others as is done classically with the utility functions in the presence of consumption externalities.

Now we describe our contributions. We first provide an example of an exchange economy with externalities and no consumption constraints where all endowments are singular and give rise to infinite equilibrium sets ${ }^{4}$. So, some restrictions on the characteristics of the agents must be made in order to get generic regularity.

In our example, externalities affect not only the utility levels but also the marginal rates of substitution. This feature does not appear if one considers additively separable utility functions as in Crès (1996) and in Geanakoplos and Polemarchakis (2008), or a more general form of separable utility functions as in Heidhues and Riedel (2007). In these latter cases, the genericity of regular economies is merely a consequence of classical results, since the equilibrium set coincides with the one of an associated economy without externalities. Indeed, for utility functions which are additively separable or à la Heidhues and Riedel, the demand does not depend on the externalities, since preferences satisfy the following independence property: if household $h$ prefers $x_{h}$ to $x_{h}^{\prime}$ for a given consumption of the others, then he always prefers $x_{h}$ to $x_{h}^{\prime}$ whatever is the consumption of the others. Consequently, there is no way to provide evidence of externalities or to measure their impact by observing market's outcomes or individual demands. But, from an economic point of view, it is important to go beyond this setting. Indeed, the literature on externalities, in particular on network externalities or social preferences, reports that the economic behavior of the agents is modified by a change of the environment. Thus, it implies that externalities have an effect on the marginal rates of substitution or on the willingness to pay for some commodities.

Our example shows that regularity fails since the external effects are too strong. So, to get generic regularity, in Assumption 9 (see Section 4) we assume that the second order external effects (consumption of the others) on both utility and possibility functions are dominated by the second order direct effect (own individual consumption). This assumption clearly puts some restrictions on the external effects on marginal rates of substitution but it allows us to go beyond the utility functions which are additively separable or à la Heidhues and Riedel, as illustrated in Section 4. We also note that in the absence of consumption constraints, Assumption 9 implies that the equilibrium set is smooth and that the demand functions satisfy the law of demand in a neighborhood of any equilibrium allocation.

Furthermore, externalities in the consumption sets may lead to a lack of con-

Siconolfi (1990), Polemarchakis and Siconolfi (1997), Cass, Siconolfi and Villanacci (2001), Carosi and Villanacci (2005).

${ }^{4}$ We thank Andreu Mas-Colell and Paolo Siconolfi who greatly helped us with it. 
tinuity on the equilibrium prices in the following sense. At equilibrium, if the consumption of a household is on the boundary of his consumption set, the prices reflect his marginal utilities as well as his marginal possibilities to consume. However, if the consumption is in the interior of the consumption set, equilibrium prices reflect only the marginal utilities, since locally the household is not constrained at all in terms of consumption possibilities. So a small change in endowments might drastically change the equilibrium prices. Indeed, changing slightly the endowments, some equilibrium consumptions which were on the boundary of the consumption sets may be moved toward the interior. Consequently, previous equilibrium prices, reflecting marginal utilities and marginal possibilities to consume, are far from the new ones which now reflect only marginal utilities. To overcome this discontinuity, we consider displacements of the boundaries of the consumption sets, that is, simple perturbations of the possibility functions (see Section 5).

Our main result, Theorem 12, states that almost all perturbed economies are regular. Then, in Corollary 13, we provide the generic regularity result in the space of endowments and possibility functions ${ }^{5}$.

It is perhaps surprising, but to the best of our knowledge, there are few results on regularity in the presence of externalities ${ }^{6}$. No one considers consumption constraints. In Bonnisseau (2003), the result is based on a geometric assumption on preferences. The economic interpretation of its extension to the case with consumption constraints would be unwise.

In Kung (2008), where also public goods and production are considered, no specific assumption on the utility functions is necessary. But the author has to perturb utility functions as well as all the other fundamentals of the economy. As noticed by the author, as many parameters as the number of the equations are needed. The smaller is the number of parameters, the stronger is the genericity result. In this trade off, we choose to use the classical parameters, that is, initial endowments, at the cost of an additional assumption on the utility functions. Indeed, in the absence of consumption constraints, economies are regular for almost all initial endowments, without perturbing any other fundamentals of the economy (see Section 5). So, our approach sheds some light on the fact that the generic regularity holds true with external effects affecting marginal rates of substitution and thus individual demands, so long as these effects are not too strong.

5 Following Mas-Colell (1985), generic means in an open and dense subset. Following Smale (1981), almost all means in an open and full measure subset.

6 In Crès (1996), Geanakoplos and Polemarchakis (2008) and Heidhues and Riedel (2007), as already mentioned, the regularity is merely a consequence of classical results. However, the purpose of these papers is not the study of regularity, since it is not an issue due to their assumptions. 
The paper is organized as follows. Section 2 is devoted to the model and basic assumptions. In Section 3, we present the definitions of competitive equilibria and of the equilibrium function. Theorem 8 recalls non-emptiness and compactness results. In Section 4, we provide an example, then we state Assumption 9 and compare it with assumptions previously made in the literature. In Section 5, we state the definitions of a regular economy and of a perturbed economy. In Section 6, we present the main results of this paper, Theorem 12 and Corollary 13. In Section 7, we prove our main results. Finally, all the lemmas are proved in Appendix A. In Appendix B, the reader can find classical results from differential topology used in our analysis.

\section{Model and basic assumptions}

There is a finite number $C$ of physical commodities labeled by the superscript $c \in\{1, \ldots, C\}$. The commodity space is $\mathbb{R}_{++}^{C}$. There is a finite number $H$ of households labeled by subscript $h \in \mathcal{H}:=\{1, \ldots, H\}$. Each household $h \in \mathcal{H}$ is characterized by an endowment of commodities, a possibility function and a utility function. Possibility and utility functions depend on the consumptions of all households.

The notations are summarized below.

- $x_{h}^{c}$ is the consumption of commodity $c$ of household $h ; x_{h}:=\left(x_{h}^{1}, . ., x_{h}^{c}, . ., x_{h}^{C}\right)$ denotes household $h$ 's consumption and $x_{-h}:=\left(x_{k}\right)_{k \neq h}$ the consumptions of households other than $h$ called the environment of household $h ; x:=$ $\left(x_{h}\right)_{h \in \mathcal{H}}$.

- $e_{h}^{c}$ is the endowment of commodity $c$ of household $h ; e_{h}:=\left(e_{h}^{1}, . ., e_{h}^{c}, . ., e_{h}^{C}\right)$ denotes household $h$ 's endowment; $e:=\left(e_{h}\right)_{h \in \mathcal{H}}$.

- As in general equilibrium model à la Arrow-Debreu, each household $h$ has to choose a consumption in his consumption set $X_{h}$, i.e., in the set of all consumption alternatives which are a priori possible for him. In the spirit of Smale's work (1974), the consumption set of household $h$ is described in terms of an inequality on a function $\chi_{h}{ }^{7}$. We call $\chi_{h}$ the possibility function.

The main innovation of this paper comes from the dependency of the consumption set of each household with respect to the consumptions of the other households, i.e., given $x_{-h} \in \mathbb{R}_{++}^{C(H-1)}$, the consumption set of

\footnotetext{
$\overline{7}$ Observe that this idea is usual for smooth economies with production where each production set is described by an inequality on a function called the transformation function, see Villanacci et al. (2002), for instance.
} 
household $h$ is the following set $^{8}$,

$$
X_{h}\left(x_{-h}\right)=\left\{x_{h} \in \mathbb{R}_{++}^{C}: \chi_{h}\left(x_{h}, x_{-h}\right) \geq 0\right\}
$$

where the possibility function $\chi_{h}$ is a function from $\mathbb{R}_{++}^{C} \times \mathbb{R}_{+}^{C(H-1)}$ to $\mathbb{R}$.

- Each household $h \in \mathcal{H}$ has preferences described by a utility function $u_{h}$ from $\mathbb{R}_{++}^{C} \times \mathbb{R}_{+}^{C(H-1)}$ to $\mathbb{R}$, and $u_{h}\left(x_{h}, x_{-h}\right)$ is household $h$ 's utility level associated with the consumption $x_{h}$ and the environment $x_{-h}$.

- $\left(u_{h}, \chi_{h}, e_{h}\right)_{h \in \mathcal{H}}$ is an economy.

- $p^{c} \in \mathbb{R}_{++}$is the price of one unit of commodity $c ; p:=\left(p^{1}, . ., p^{c}, . ., p^{C}\right) \in$ $\mathbb{R}_{++}^{C}$.

- Given a vector $w=\left(w^{1}, . ., w^{c}, . ., w^{C}\right) \in \mathbb{R}^{C}$, we denote $w^{\backslash}:=\left(w^{1}, . ., w^{c}, . ., w^{C-1}\right) \in \mathbb{R}^{C-1}$.

From now on we make the following assumptions on utility and possibility functions taken from del Mercato $(2006)^{9}$.

Assumption 1 For all $h \in \mathcal{H}$,

(1) $u_{h}$ is continuous on $\mathbb{R}_{++}^{C} \times \mathbb{R}_{+}^{C(H-1)}$ and $C^{2}$ in the interior of its domain.

(2) for each $x_{-h} \in \mathbb{R}_{++}^{C(H-1)}$, the function $u_{h}\left(\cdot, x_{-h}\right)$ is differentiably strictly increasing, i.e., for every $x_{h} \in \mathbb{R}_{++}^{C}, D_{x_{h}} u_{h}\left(x_{h}, x_{-h}\right) \in \mathbb{R}_{++}^{C}$.

(3) For each $x_{-h} \in \mathbb{R}_{++}^{C(H-1)}$, the function $u_{h}\left(\cdot, x_{-h}\right)$ is differentiably strictly quasi-concave, i.e., for every $x_{h} \in \mathbb{R}_{++}^{C}, D_{x_{h}}^{2} u_{h}\left(x_{h}, x_{-h}\right)$ is negative definite on $\operatorname{Ker} D_{x_{h}} u_{h}\left(x_{h}, x_{-h}\right)^{10}$.

(4) For each $x_{-h} \in \mathbb{R}_{+}^{C(H-1)}$ and for every $u \in \operatorname{Im} u_{h}, c l_{\mathbb{R}^{C}}\left\{x_{h} \in \mathbb{R}_{++}^{C}\right.$ : $\left.u_{h}\left(x_{h}, x_{-h}\right) \geq u\right\} \subseteq \mathbb{R}_{++}^{C}$.

Assumption 2 For all $h \in \mathcal{H}$,

(1) $\chi_{h}$ is continuous on $\mathbb{R}_{++}^{C} \times \mathbb{R}_{+}^{C(H-1)}$ and $C^{2}$ in the interior of its domain.

(2) (Convexity of the consumption set) For each $x_{-h} \in \mathbb{R}_{+}^{C(H-1)}$, the function

8 Note that in Smale (1974), each consumption set is described by several inequality constraints. Our results can be extended to this case, but this is not our main objective.

9 In this paper, possibility functions also depend on endowments. For the sake of clarity, here we consider only externalities.

${ }^{10}$ Let $v$ and $v^{\prime}$ be two vectors in $\mathbb{R}^{n}, v \cdot v^{\prime}$ denotes the inner product of $v$ and $v^{\prime}$. Let $A$ be a real matrix with $m$ rows and $n$ columns, and $B$ be a real matrix with $n$ rows and $l$ columns, $A B$ denotes the matrix product of $A$ and $B$. Without loss of generality, vectors are treated as row matrices and $A$ denotes both the matrix and the following linear application $A: v \in \mathbb{R}^{n} \rightarrow A(v):=A v^{T} \in \mathbb{R}^{[m]}$ where $v^{T}$ denotes the transpose of $v$ and $\mathbb{R}^{[m]}:=\left\{w^{T}: w \in \mathbb{R}^{m}\right\}$. When $m=1, A(v)$ coincides with the inner product $A \cdot v$, treating $A$ and $v$ as vectors in $\mathbb{R}^{n}$. 
$\chi_{h}\left(\cdot, x_{-h}\right)$ is quasi-concave ${ }^{11}$.

(3) (Survival condition) There exists $\bar{x}_{h} \in \mathbb{R}_{++}^{C}$ such that $\chi_{h}\left(\bar{x}_{h}, x_{-h}\right) \geq 0$ for every $x_{-h} \in \mathbb{R}_{+}^{C(H-1)}$.

(4) (Non-satiation) For each $x_{-h} \in \mathbb{R}_{++}^{C(H-1)}$ and for every $x_{h} \in \mathbb{R}_{++}^{C}$,

(a) $D_{x_{h}} \chi_{h}\left(x_{h}, x_{-h}\right) \neq 0$; (b) $D_{x_{h}} \chi_{h}\left(x_{h}, x_{-h}\right) \notin-\mathbb{R}_{++}^{C}$.

(5) (Global desirability) For each $x \in \mathbb{R}_{++}^{C H}$ and for each $c \in\{1, \ldots, C\}$ there exists $h(c) \in \mathcal{H}$ such that $D_{x_{h(c)}^{c}} \chi_{h(c)}\left(x_{h(c)}, x_{-h(c)}\right) \in \mathbb{R}_{+}$.

For the interpretation of Points 1, 2, 4 and 5 of Assumption 2 we refer to del Mercato (2006), pp. 529-530.

Point 3 of Assumption 2 is called the "survival condition" since it guarantees that there exists a consumption belonging to the consumption set $X_{h}\left(x_{-h}\right)$ of household $h$, whatever are the consumptions $x_{-h} \in \mathbb{R}_{++}^{C(H-1)}$ of the others.

Note that in order to get compactness and properness properties of the equilibrium set, in Points 1 and 4 of Assumption 1 and in Points 1, 2 and 3 of Assumption 2, we consider consumptions $x_{-h}$ in the closure of $\mathbb{R}_{++}^{C(H-1)}$. This gives some information on the boundary behavior of $u_{h}$ and $\chi_{h}$.

Definition $3 \mathcal{U}$ denotes the set of $u=\left(u_{h}\right)_{h \in \mathcal{H}}$ which satisfies Assumption 1, and $\mathcal{X}$ denotes the set of $\chi=\left(\chi_{h}\right)_{h \in \mathcal{H}}$ which satisfies Assumption 2.

We now define the set of endowments, which satisfy the survival assumption for the given possibility functions. That is, endowments such that for each household there exists in his consumption set an interior consumption which is strictly smaller than his endowments.

Definition 4 Let $\chi \in \mathcal{X}$. Define the set $E_{\chi}:=\prod_{h \in \mathcal{H}} E_{\chi_{h}} \subseteq \mathbb{R}_{++}^{C H}$ where

$$
E_{\chi_{h}}:=\left(\bigcap_{x_{-h} \in \mathbb{R}_{+}^{C(H-1)}}\left\{x_{h} \in \mathbb{R}_{++}^{C}: \chi_{h}\left(x_{h}, x_{-h}\right) \geq 0\right\}\right)+\mathbb{R}_{++}^{C}
$$

From Point 3 of Assumption 2, $E_{\chi}$ is nonempty and it is open by definition. The survival assumption (Point 3 of Assumption 2 in del Mercato, 2006) is satisfied on $E_{\chi}$, that is, for all $e=\left(e_{h}\right)_{h \in \mathcal{H}} \in E_{\chi}$ and for every $h \in \mathcal{H}$ :

$$
\forall x_{-h} \in \mathbb{R}_{+}^{C(H-1)}, \exists \widetilde{x}_{h} \in \mathbb{R}_{++}^{C}: \chi_{h}\left(\widetilde{x}_{h}, x_{-h}\right)>0 \text { and } \widetilde{x}_{h} \ll e_{h}
$$

Since $\chi_{h}$ is $C^{2}$ in the interior of its domain, then for each $x_{-h} \in \mathbb{R}_{++}^{C(H-1)}$, the function $\chi_{h}\left(\cdot, x_{-h}\right)$ is differentiably quasi-concave, i.e., and for every $x_{h} \in \mathbb{R}_{++}^{C}$, $D_{x_{h}}^{2} \chi_{h}\left(x_{h}, x_{-h}\right)$ is negative semidefinite on $\operatorname{Ker} D_{x_{h}} \chi_{h}\left(x_{h}, x_{-h}\right)$. 
Remark 5 From now on, $u \in \mathcal{U}$ is kept fixed and an economy is parameterized by $(\chi, e)$ taken in the following set.

$$
\Theta:=\left\{(\chi, e) \in\left(C^{0-2}(T, \mathbb{R})\right)^{H} \times \mathbb{R}_{++}^{C H}: \chi \in \mathcal{X} \text { and } e \in E_{\chi}\right\}
$$

where $T:=\mathbb{R}_{++}^{C} \times \mathbb{R}_{+}^{C(H-1)}$ and $C^{0-2}(T, \mathbb{R})$ is defined by (20) in Appendix $B$.

\section{Competitive equilibrium with externalities}

The definitions and the results stated in this section are direct transpositions of the ones in del Mercato (2006).

Without loss of generality, commodity $C$ is the numéraire good. Then, given $p \backslash \in \mathbb{R}_{++}^{C-1}$ with innocuous abuse of notation we denote $p:=(p \backslash, 1) \in \mathbb{R}_{++}^{C}$.

Definition $6\left(x^{*}, p^{*}\right) \in \mathbb{R}_{++}^{C H} \times \mathbb{R}_{++}^{C-1}$ is a competitive equilibrium for the economy $(\chi, e)$ if for all $h \in \mathcal{H}, x_{h}^{*}$ solves the following problem

$$
\begin{array}{ll}
\max _{x_{h} \in \mathbb{R}_{++}^{C}} & u_{h}\left(x_{h}, x_{-h}^{*}\right) \\
\text { subject to } & \chi_{h}\left(x_{h}, x_{-h}^{*}\right) \geq 0 \\
& p^{*} \cdot x_{h} \leq p^{*} \cdot e_{h}
\end{array}
$$

and $x^{*}$ satisfies market clearing conditions

$$
\sum_{h \in \mathcal{H}} x_{h}^{*}=\sum_{h \in \mathcal{H}} e_{h}
$$

From now on we follow Smale's extended approach, that is, an equilibrium is defined as a zero of an equilibrium function explicitly built with the KuhnTucker conditions ${ }^{12}$. In the presence of externalities, this approach avoids the following effect: the individual demand functions depend on the individual demand functions of the others, which depend on the individual demand functions of the others, and so on. So, it would be impossible to define an aggregate excess demand function which depends only on prices and endowments.

Proposition 7 Let $(\chi, e) \in \Theta$ be an economy, $x_{-h}^{*} \in \mathbb{R}_{++}^{C(H-1)}$ and $p^{* \backslash} \in$ $\mathbb{R}_{++}^{C-1}$. Problem (3) has a unique solution. $x_{h}^{*} \in \mathbb{R}_{++}^{C}$ is the solution to problem

\footnotetext{
${ }^{12}$ See Smale $(1974,1981)$. The reader can also find a survey of this approach in Villanacci et al. (2002).
} 
(3) if and only if there exists $\left(\lambda_{h}^{*}, \mu_{h}^{*}\right) \in \mathbb{R}_{++} \times \mathbb{R}$ such that $\left(x_{h}^{*}, \lambda_{h}^{*}, \mu_{h}^{*}\right)$ is the unique solution of the following system.

$$
\left\{\begin{array}{l}
(h .1) D_{x_{h}} u_{h}\left(x_{h}, x_{-h}^{*}\right)-\lambda_{h} p+\mu_{h} D_{x_{h}} \chi_{h}\left(x_{h}, x_{-h}^{*}\right)=0 \\
(h .2)-p^{*} \cdot\left(x_{h}-e_{h}\right)=0 \\
(h .3) \min \left\{\mu_{h}, \chi_{h}\left(x_{h}, x_{-h}^{*}\right)\right\}=0
\end{array}\right.
$$

Define the set of endogenous variables as $\Xi:=\left(\mathbb{R}_{++}^{C} \times \mathbb{R}_{++} \times \mathbb{R}\right)^{H} \times \mathbb{R}_{++}^{C-1}$, with generic element $\xi:=\left(x, \lambda, \mu, p^{\backslash}\right):=\left(\left(x_{h}, \lambda_{h}, \mu_{h}\right)_{h \in \mathcal{H}}, p^{\backslash}\right)$. We can now describe extended equilibria using system (5) and market clearing conditions (4). Observe that, from Definition 6 and Proposition 7, the market clearing condition for good $C$ is "redundant" (see equations $(h .2)_{h \in \mathcal{H}}$ in (5)). The equilibrium function defined below takes into account this aspect. For each economy $(\chi, e) \in \Theta$, the equilibrium function $F_{\chi, e}: \Xi \rightarrow \mathbb{R}^{\operatorname{dim} \Xi}$

$$
F_{\chi, e}(\xi):=\left(\left(F_{\chi, e}^{h .1}(\xi), F_{\chi, e}^{h .2}(\xi), F_{\chi, e}^{h .3}(\xi)\right)_{h \in \mathcal{H}}, F_{\chi, e}^{M}(\xi)\right)
$$

is defined by

$F_{\chi, e}^{h .1}(\xi):=D_{x_{h}} u_{h}\left(x_{h}, x_{-h}\right)-\lambda_{h} p+\mu_{h} D_{x_{h}} \chi_{h}\left(x_{h}, x_{-h}\right), F_{\chi, e}^{h .2}(\xi):=-p \cdot\left(x_{h}-e_{h}\right)$, $F_{\chi, e}^{h .1}(\xi):=\min \left\{\mu_{h}, \chi_{h}\left(x_{h}, x_{-h}\right)\right\}$, and $F_{\chi, e}^{M}(\xi):=\sum_{h \in \mathcal{H}}\left(x_{h}^{\curlywedge}-e_{h}^{\backslash}\right)$.

$\xi^{*} \in \Xi$ is an extended competitive equilibrium (also called with an innocuous abuse of terminology equilibrium) of the economy $(\chi, e) \in \Theta$ if $F_{\chi, e}\left(\xi^{*}\right)=0$.

Theorem 8 (Existence and compactness). For each economy $(\chi, e) \in \Theta$, the equilibrium set $F_{\chi, e}^{-1}(0)$ is non-empty and compact.

\section{An example and an additional assumption}

To illustrate the fact that the two previous assumptions are not sufficient to get generic regularity, we consider the following example. Consider a two commodity-two household economy with consumption sets coinciding with the whole commodity space $\mathbb{R}_{++}^{2}$ and with the following utility functions.

$$
u_{1}\left(x_{1}, x_{2}\right):=\ln \left((1+\varepsilon) x_{1}^{1}+x_{2}^{1}\right)+x_{1}^{2}+\frac{1}{1+\varepsilon} x_{2}^{2}:=u_{2}\left(x_{2}, x_{1}\right), \text { with } \varepsilon>0
$$


One easily checks that for each $\left(e_{1}, e_{2}\right) \in\left(\mathbb{R}_{++}^{2}\right)^{2}$,

$$
\left(\left(e_{1}^{1}-t_{1}, e_{1}^{2}+t_{2}\right),\left(e_{2}^{1}+t_{1}, e_{2}^{2}-t_{2}\right), p^{* 1}=\frac{1+\varepsilon}{\left(e_{1}^{1}+e_{2}^{1}\right)+\varepsilon\left(e_{1}^{1}-t_{1}\right)}\right)
$$

is an equilibrium for every $\left(t_{1}, t_{2}\right) \in \mathbb{R}^{2}$ such that $t_{1}$ belongs to an appropriate neighborhood of 0 and $t_{2}=t_{1} p^{* 1}$. So, no economy $\left(e_{1}, e_{2}\right) \in\left(\mathbb{R}_{++}^{2}\right)^{2}$ has a finite number of equilibria, which implies that all economies are singular.

This phenomenon can be explained by the fact that the external effect of household 1 on household 2 is too strong with respect to the effect of household 2 's own consumption. Indeed, let us consider the marginal rate of substitution $\operatorname{MRS}_{2}\left(x_{1}, x_{2}\right)$ of household 2 at $\left(x_{1}, x_{2}\right)$, which is equal to $\frac{(1+\varepsilon)}{(1+\varepsilon) x_{1}^{1}+x_{2}^{1}}$. So $\left|\frac{\partial \mathrm{MRS}_{2}}{\partial x_{1}^{1}}\left(x_{1}, x_{2}\right)\right|=\frac{(1+\varepsilon)^{2}}{\left((1+\varepsilon) x_{1}^{1}+x_{2}^{1}\right)^{2}}>\left|\frac{\partial \mathrm{MRS}_{2}}{\partial x_{2}^{1}}\left(x_{1}, x_{2}\right)\right|=\frac{(1+\varepsilon)}{\left((1+\varepsilon) x_{1}^{1}+x_{2}^{1}\right)^{2}}$

Thus, we introduce the following additional assumption.

Assumption 9 Let $(x, v) \in \mathbb{R}_{++}^{C H} \times \mathbb{R}^{C H}$ such that $v \in \prod_{h \in \mathcal{H}} \operatorname{Ker} D_{x_{h}} u_{h}\left(x_{h}, x_{-h}\right)$ and $\sum_{h \in \mathcal{H}} v_{h}=0$. Then,

(1) $v_{h} \sum_{k \in \mathcal{H}} D_{x_{k} x_{h}}^{2} u_{h}\left(x_{h}, x_{-h}\right)\left(v_{k}\right)<0$ whenever $v_{h} \neq 0$, and

(2) $v_{h} \sum_{k \in \mathcal{H}} D_{x_{k} x_{h}}^{2} \chi_{h}\left(x_{h}, x_{-h}\right)\left(v_{k}\right) \leq 0$ whenever $v_{h} \in \operatorname{Ker} D_{x_{h}} \chi_{h}\left(x_{h}, x_{-h}\right)$.

Point 1 of Assumption 9 means that the effect of changes in the consumptions $\left(x_{k}\right)_{k \neq h}$ of households other than $h$ on the marginal utility $D_{x_{h}} u_{h}\left(x_{h}, x_{-h}\right)$ is "dominated" by the effect of changes in the consumption $x_{h}$ of household $h$. Indeed, under Point 3 of Assumption 1, Point 1 of Assumption 9 states that the absolute value of $v_{h} D_{x_{h}}^{2} u_{h}\left(x_{h}, x_{-h}\right)\left(v_{h}\right)$ is larger than the remaining term $v_{h} \sum_{k \neq h} D_{x_{k} x_{h}}^{2} u_{h}\left(x_{h}, x_{-h}\right)\left(v_{k}\right)$.

Under Points $1 b$ and 2 of Assumption 2, Point 2 of Assumption 9 has the same interpretation as Point 1 for the possibility functions.

We can give a more precise statement when the externalities are almost uniform in the sense that the orthogonal projection on $\operatorname{Ker} D_{x_{h}} u_{h}\left(x_{h}, x_{-h}\right)$ of $D_{x_{k} x_{h}}^{2} u_{h}\left(x_{h}, x_{-h}\right)$ does not depend on $k$ for all $k \neq h$. Indeed, let us denote by $D_{y x_{h}}^{2}$ proj $\circ u_{h}\left(x_{h}, x_{-h}\right)$ the matrix of the composition of the projection and 


$$
\begin{aligned}
& D_{x_{k} x_{h}}^{2} u_{h}\left(x_{h}, x_{-h}\right) . \text { Then, since } \sum_{k \in \mathcal{H}} v_{k}=0 \text { and } v_{h} \in \operatorname{Ker} D_{x_{h}} u_{h}\left(\left(x_{h}, x_{-h}\right),\right. \\
& v_{h} \sum_{k \in \mathcal{H}} D_{x_{k} x_{h}}^{2} u_{h}\left(x_{h}, x_{-h}\right)\left(v_{k}\right)= v_{h}\left(D_{x_{h} x_{h}}^{2} u_{h}\left(x_{h}, x_{-h}\right)-\right. \\
&\left.D_{y x_{h}}^{2} \operatorname{proj} \circ u_{h}\left(x_{h}, x_{-h}\right)\right)\left(v_{h}\right) .
\end{aligned}
$$

So, Assumption 9(1) holds true if the matrix $D_{x_{h} x_{h}}^{2} u_{h}\left(x_{h}, x_{-h}\right)-D_{y x_{h}}^{2}$ proj ○ $u_{h}\left(x_{h}, x_{-h}\right)$ is negative definite on $\operatorname{Ker} D_{x_{h}} u_{h}\left(x_{h}, x_{-h}\right)$. Since we already know that the matrix $D_{x_{h} x_{h}}^{2} u_{h}\left(x_{h}, x_{-h}\right)$ is negative definite on $\operatorname{Ker} D_{x_{h}} u_{h}\left(x_{h}, x_{-h}\right)$ by Point 3 of Assumption 1, we get the result if the norm of $D_{y x_{h}}^{2} u_{h}\left(x_{h}, x_{-h}\right)$ is small enough with respect to the norm of $D_{x_{h} x_{h}}^{2} u_{h}\left(x_{h}, x_{-h}\right)$, which means that the external effect is small with respect to the direct effect of the consumption of the household. For example, let $w_{h}$ be a usual utility function on $\mathbb{R}_{++}^{C}$ satisfying the standard differentiability assumption and $u_{h}$ be defined by:

$$
u_{h}\left(x_{h}, x_{-h}\right)=w_{h}\left(x_{h}+\rho \sum_{k \neq h} x_{k}\right)
$$

For $\rho>0$ strictly smaller than 1, Assumption 9(1) is satisfied.

In the absence of consumption constraints, we can also deduce two important properties of the economies satisfying Assumption 9(1). Let us define the set of supported allocations for a price $p \in \mathbb{R}_{++}^{C}$ as follows:

$$
S(p)=\left\{x \in \mathbb{R}_{++}^{C H} \mid \forall h \in \mathcal{H}, \exists \lambda_{h}>0, D_{x_{h}} u_{h}\left(x_{h}, x_{-h}\right)=\lambda_{h} p\right\}
$$

We can check that the proof of the main theorem works identically if we only put Assumption 9 on the set of consumptions in $\cup_{p \in \mathbb{R}_{++}^{C}} S(p)$. For given initial endowments $e$, let us define the set of equilibrium points as follows:

$$
\operatorname{Eq}(e)=\left\{x \in \mathbb{R}_{++}^{C H} \mid \sum_{h \in \mathcal{H}} x_{h}-e_{h}=0, \exists p \in \mathbb{R}_{++}^{C}, x \in S(p)\right\}
$$

We remark that without externalities, an equilibrium allocation always belongs to the set $\mathrm{Eq}(\mathrm{e})$, which is also called contract curve. We could also call it the set of no-trade equilibria since if the initial endowments belong to this set, then there exists an equilibrium where all agents keep their initial endowments. This set is the set of Pareto optimal allocations if there are no externalities.

Using the same tools as in the proof of the main theorem, we can show that the set $\operatorname{Eq}(e)$ is a manifold of dimension $C-1$ under Assumption 9 (1). So we recover an important property of the equilibrium set that is known for economies without externalities.

Let us now consider the endowments $e$ such that $e \in S(\bar{p})$ for some $\bar{p} \in \mathbb{R}_{++}^{C}$. 
Let us define the global demand $\delta(p, e)$ as follows:

$$
\delta(p, e)=\left\{x \in S(p) \mid \forall h \in \mathcal{H}, p \cdot x_{h}=p \cdot e_{h}\right\}
$$

Assumption 9 implies that $\delta(\cdot, e)$ is a differentiable mapping on a neighborhood of $\bar{p}$. Furthermore, the demand satisfies the law of demand at $\bar{p}$, that is $D_{p} \delta_{h}(\bar{p}, e)(\Delta p) \cdot \Delta p<0$ for all vectors $\Delta p$ orthogonal to $p$. In the standard case without externalities, this property is deduced from the fact that the Slutsky matrix is negative definite on the orthogonal complement of $p$. So, Assumption 9 implies that the law of demand remains true in the absence of the revenue effect.

\subsection{Comparison with previous assumptions}

If the external effects do not influence the supporting prices of any allocation, that is $D_{x_{h}} u_{h}\left(x_{h}, x_{-h}\right)$ and $D_{x_{h}} \chi_{h}\left(x_{h}, x_{-h}\right)$ do not depend on $x_{-h}$, then under Assumptions 1 and 2, Assumption 9 is clearly satisfied since $D_{x_{k} x_{h}}^{2} u_{h}\left(x_{h}, x_{-h}\right)=0$ and $D_{x_{k} x_{h}}^{2} \chi_{h}\left(x_{h}, x_{-h}\right)=0$ for every $k \neq h$. This is the case of Smale (1974) with no externalities at all, and of Crès (1996) and Geanakoplos and Polemarchakis (2008) where utility functions are additively separable between individual consumption and external effects.

In Heidhues and Riedel (2007), the authors consider a more general functional form of separable utility functions, but Assumption 9 is still valid since the range of $D_{x_{k} x_{h}}^{2} u_{h}\left(x_{h}, x_{-h}\right)$ is included in the line generated by $D_{x_{h}} u_{h}\left(x_{h}, x_{-h}\right)$. We also remark that the example of utility function given in (7) does not satisfy the Heidhues and Riedel's assumption since the derivative of $u_{h}$ does not remain collinear with respect to the consumptions $x_{-h}$.

However, the objective of these papers is not the genericity of regular economies, since they are dealing with Pareto optimality issues or equivalence of the equilibrium set with and without externalities.

In Kung (2008), utility functions satisfy assumptions similar to the ones given in Assumption 1. There are no additional assumption on the utility functions but the author needs to perturb the utility functions to obtain generic regularity in the class of economy he considers, as widely discussed in Introduction.

In Bonnisseau (2003), preferences are more general than the ones considered in this paper, since they are non transitive and non complete. In this general setting, the author obtains the result of regularity for almost all endowments, under a geometric assumption. To borrow this assumption in our framework with possibility functions, it would be necessary to involve the Lagrange multipliers associated with the possibility functions. Consequently, utility and 
possibility functions would not be considered independently. So, the economic interpretation would be unwise.

\section{Regular economies and possibility perturbations}

Let us start with the definition of regular economy.

Definition $10(\chi, e) \in \Theta$ is a regular economy if for each $\xi^{*} \in F_{\chi, e}^{-1}(0)$,

(1) $F_{\chi, e}$ is a $C^{1}$ function around $\xi^{* 13}$.

(2) The differential mapping $D_{\xi} F_{\chi, e}\left(\xi^{*}\right)$ is onto.

Our analysis is based on results from differential topology, in the spirit of the works of Balasko, Debreu, Mas-Colell and Smale. Since nothing prevents the equilibrium consumptions from being on the boundaries of the consumption sets, for every $h \in \mathcal{H}$ the function $F_{\chi, e}^{h .3}(\xi)=\min \left\{\mu_{h}, \chi_{h}\left(x_{h}, x_{-h}\right)\right\}$ is not $C^{1}$ if $\mu_{h}=0$ and $\chi_{h}\left(x_{h}, x_{-h}\right)=0$. Therefore, first of all, it shall be shown that this case is exceptional at each equilibrium. For that purpose we follow the strategy laid out in Cass, Siconolfi and Villanacci (2001), where general portfolio sets are encompassed while still permitting differentiable techniques.

But, the presence of externalities in the consumption sets leads to a possible lack of continuity in the set of household's supporting prices. Indeed, consider the situation in which, at equilibrium, the consumption of household $h$ is on the boundary of his consumption set, i.e., $\chi_{h}\left(x_{h}^{*}, x_{-h}^{*}\right)=0$. If the associated multiplier $\mu_{h}^{*}$ is strictly positive, household $h$ 's supporting prices belong to the cone positively generated by $D_{x_{h}} u_{h}\left(x_{h}^{*}, x_{-h}^{*}\right)$ and $D_{x_{h}} \chi_{h}\left(x_{h}^{*}, x_{-h}^{*}\right)$. If, in every neighborhood of $x_{-h}^{*}$, households other than $h$ can move to $x_{-h}$ in such a way that $x_{h}^{*}$ is now in the interior of the consumption set, that is $\chi_{h}\left(x_{h}^{*}, x_{-h}\right)>0$, then the positive cone of household $h$ 's supporting prices collapses into the positive half-line generated by $D_{x_{h}} u_{h}\left(x_{h}^{*}, x_{-h}\right)$. If the associated multiplier $\mu_{h}^{*}$ is equal to 0 , household $h$ 's supporting prices belong to the positive half-line generated by $D_{x_{h}} u_{h}\left(x_{h}^{*}, x_{-h}^{*}\right)$. If, in every neighborhood of $x_{-h}^{*}$, households other than $h$ can move to $x_{-h}$ in such a way that $x_{h}^{*}$ remains on the boundary of the consumption set, i.e., $\chi_{h}\left(x_{h}^{*}, x_{-h}\right)=0$, then the positive half-line of household $h$ 's supporting prices might spread over the cone positively generated by $D_{x_{h}} u_{h}\left(x_{h}^{*}, x_{-h}\right)$ and $D_{x_{h}} \chi_{h}\left(x_{h}^{*}, x_{-h}\right)$. In both cases, previous household $h$ 's supporting prices may be far from the new ones.

Therefore, we consider displacements of the boundaries of the consumption sets, that is, simple perturbations of the possibility functions. The following is

$\overline{{ }^{13} F_{\chi, e}}$ is a $C^{1}$ function around $\xi^{*}$ means that there exists an open neighborhood $I\left(\xi^{*}\right)$ of $\xi^{*}$ in $\Xi$ such that the restriction of $F_{\chi, e}$ to $I\left(\xi^{*}\right)$ is a $C^{1}$ function. 
the definition of a perturbed economy for a given $\chi \in \mathcal{X}$.

Definition 11 A perturbed economy $(\chi+a, e)$ is parameterized by possibility levels $a=\left(a_{h}\right)_{h \in \mathcal{H}} \in \mathbb{R}_{++}^{H}$ and endowments $e \in E_{\chi}$, and it is defined by

$$
\chi+a:=\left(\chi_{h}+a_{h}\right)_{h \in \mathcal{H}}
$$

$\Lambda_{\chi}:=\mathbb{R}_{++}^{H} \times E_{\chi}$ denotes the set of perturbed economies.

It is an easy matter to check that for every $(a, e) \in \Lambda_{\chi}$, the perturbed economy $(\chi+a, e) \in \Theta$.

Finally, note that for the reasons mentioned above:

(1) if all consumption sets coincide with $\mathbb{R}_{++}^{C}$, i.e., for all $h \in \mathcal{H}, X_{h}\left(x_{-h}\right)=$ $\mathbb{R}_{++}^{C}$ for any given $x_{-h}$, then perturbations of the possibility functions are not needed. So, under Point 1 of Assumption 9, the result of regular economies holds true for almost all initial endowments.

(2) if utility and possibility functions do not depend on the environment, as in Smale (1974), then, once again, perturbations of the possibility functions are not needed. Indeed, the set of household $h$ 's supporting prices does not depend on the consumptions of the others.

\section{Main results}

We now state the main results of the paper: the regularity for almost all perturbed economies and the generic regularity in the space of endowments and possibility functions.

Theorem 12 (Regularity for almost all perturbed economies). Let $\chi \in \mathcal{X}$. The set $\Lambda_{\chi}^{r}$ of $(a, e) \in \Lambda_{\chi}$ such that $(\chi+a, e)$ is a regular economy is an open and full measure subset of $\Lambda_{\chi}$.

Now, endow the set $C^{0-2}(T, \mathbb{R})$ with the topology of the $C^{0-2}$ uniform convergence on compacta (see Definition 21 in Appendix B), the set $\mathbb{R}_{++}^{C H}$ with the topology induced by the usual topology on $\mathbb{R}^{C H}$, and the set $\Theta$ with the topology induced by the product topology on $\left(C^{0-2}(T, \mathbb{R})\right)^{H} \times \mathbb{R}_{++}^{C H}$. As a consequence of Theorem 12 we obtain the following corollary.

Corollary 13 (Generic regularity). The set $\mathcal{R}$ of $(\chi, e) \in \Theta$ such that $(\chi, e)$ is a regular economy is an open and dense subset of $\Theta$.

From Corollary 13, Theorem 8, a consequence of the Regular Value Theorem, and the Implicit Function Theorem (see Corollary 25 and Theorem 28 in 
Appendix B), we obtain the following proposition which provides the main properties of regular economies.

Proposition 14 (Properties of regular economies). For each $(\chi, e) \in \mathcal{R}$,

(1) the equilibrium set associated with the economy $(\chi, e)$ is a non-empty finite set, i.e.,

$$
\exists r \in \mathbb{N} \backslash\{0\}: F_{\chi, e}^{-1}(0)=\left\{\xi^{1}, \ldots, \xi^{r}\right\}
$$

(2) there exist an open neighborhood I of $(\chi, e)$ in $\Theta$, and for each $i=1, \ldots, r$ an open neighborhood $N_{i}$ of $\xi^{i}$ in $\Xi$ and a continuous function $g_{i}: I \rightarrow N_{i}$ such that

(a) $N_{j} \cap N_{k}=\emptyset$ if $j \neq k$,

(b) $g_{i}(\chi, e)=\xi^{i}$,

(c) for all $\left(\chi^{\prime}, e^{\prime}\right) \in I, F_{\chi^{\prime}, e^{\prime}}^{-1}(0)=\left\{g_{i}\left(\chi^{\prime}, e^{\prime}\right): i=1, \ldots, r\right\}$,

(d) the economies $\left(\chi^{\prime}, e^{\prime}\right) \in I$ are regular.

\section{Proofs}

The proof of Theorem 12 is divided into two steps: first, we prove that the equilibrium function is $C^{1}$ around each equilibrium, for almost all perturbed economies. Second, we show that almost all perturbed economies are regular.

Corollary 13 is then deduced from Theorem 12 by using the particular form of perturbations. The proofs of the lemmas are gathered in Appendix A.

\subsection{The equilibrium function is almost everywhere $C^{1}$}

Take for fixed $\chi \in \mathcal{X}$ and consider the set of perturbed economies $\Lambda_{\chi}$ given in Definition 11. We prove the following statement.

Proposition 15 The set $\Lambda_{\chi}^{1}$ of $(a, e) \in \Lambda_{\chi}$ such that for each $\xi^{*} \in F_{\chi+a, e}^{-1}(0)$, $F_{\chi+a, e}$ is a $C^{1}$ function around $\xi^{*}$ is an open and full measure subset of $\Lambda_{\chi}$.

From Assumptions 1 and 2, the equilibrium function $F_{\chi+a, e}$ is differentiable everywhere but not at any point $\xi$ such that $\mu_{h}=\chi_{h}\left(x_{h}, x_{-h}\right)+a_{h}=0$. To prove that this so-called border line case is exceptional, we consider a finite family of auxiliary functions. We then show that a border line case is a preimage of 0 by one of these functions and that the set of perturbed economies for which the pre-image of 0 is non-empty is exceptional. 
We consider the equilibrium function $\widetilde{F}: \Xi \times \Lambda_{\chi} \rightarrow \mathbb{R}^{\operatorname{dim} \Xi}$ defined by

$$
\widetilde{F}(\xi, a, e):=F_{\chi+a, e}(\xi)
$$

We also consider the mapping $\Phi$ which is the restriction to $\widetilde{F}^{-1}(0)$ of the projection of $\Xi \times \Lambda_{\chi}$ onto $\Lambda_{\chi}$, that is:

$$
\Phi:(\xi, a, e) \in \widetilde{F}^{-1}(0) \rightarrow \Phi(\xi, a, e):=(a, e) \in \Lambda_{\chi}
$$

We state a fundamental property of $\Phi$. A similar result is proved in del Mercato (2006).

Lemma 16 The projection $\Phi: \widetilde{F}^{-1}(0) \rightarrow \Lambda_{\chi}$ is a proper function.

For all $h \in \mathcal{H}$, we consider the set

$$
\widetilde{B}_{h}:=\left\{(\xi, a, e) \in \widetilde{F}^{-1}(0): \mu_{h}=\chi_{h}\left(x_{h}, x_{-h}\right)+a_{h}=0\right\} \text { and } \widetilde{B}:=\bigcup_{h \in \mathcal{H}} \widetilde{B}_{h}
$$

We remark that

$$
\Lambda_{\chi}^{1}=\Lambda_{\chi} \backslash \Phi(\widetilde{B})
$$

Then $\Lambda_{\chi}^{1}$ is open since $\widetilde{B}$ is clearly closed in $\widetilde{F}^{-1}(0)$ and $\Phi(\widetilde{B})$ is then closed by the properness of $\Phi$.

We now show that $\Phi(\widetilde{B})$ is of measure zero in $\Lambda_{\chi}$. For this, we consider the following finite set

$$
\mathcal{P}:=\left\{\begin{array}{l|l}
\mathcal{J}=\left\{\mathcal{J}_{1}, \mathcal{J}_{2}, \mathcal{J}_{3}\right\} & \begin{array}{l}
\mathcal{J}_{i} \subseteq \mathcal{H}, \forall i=1,2,3 ; \mathcal{J}_{1} \cup \mathcal{J}_{2} \cup \mathcal{J}_{3}=\mathcal{H} ; \\
\mathcal{J}_{i} \cap \mathcal{J}_{j}=\emptyset, \forall i, j=1,2,3, i \neq j
\end{array}
\end{array}\right\}
$$

For all $\mathcal{J} \in \mathcal{P},\left|\mathcal{J}_{i}\right|$ denotes the number of elements of $\mathcal{J}_{i}$ and

$$
\Xi_{\mathcal{J}}:=\mathbb{R}_{++}^{(C+1) H} \times\left(\mathbb{R}^{\left|\mathcal{J}_{1}\right|+\left|\mathcal{J}_{3}\right|} \times \mathbb{R}_{++}^{\left|\mathcal{J}_{2}\right|}\right) \times \mathbb{R}_{++}^{(C-1)}
$$

The function $\widetilde{F}_{\mathcal{J}}: \Xi_{\mathcal{J}} \times \Lambda_{\chi} \rightarrow \mathbb{R}^{\operatorname{dim} \Xi_{\mathcal{J}}}$ is defined by

$$
\widetilde{F}_{\mathcal{J}}(\xi, a, e):=\left(\left(\widetilde{F}^{h .1}(\xi, a, e), \widetilde{F}^{h .2}(\xi, a, e), \widetilde{F}_{\mathcal{J}}^{h .3}(\xi, a, e)\right)_{h \in \mathcal{H}}, \widetilde{F}^{M}(\xi, a, e)\right)
$$

where $\widetilde{F}_{\mathcal{J}}$ differs from $\widetilde{F}$ defined in (8), for the domain and for the component $\widetilde{F}_{\mathcal{J}}^{h .3}$ defined by

$$
\widetilde{F}_{\mathcal{J}}^{h .3}(\xi, a, e):=\left\{\begin{array}{l}
\mu_{h} \text { if } h \in \mathcal{J}_{1} \cup \mathcal{J}_{3} \\
\chi_{h}\left(x_{h}, x_{-h}\right)+a_{h} \text { if } h \in \mathcal{J}_{2}
\end{array}\right.
$$


When $\mathcal{J}_{3}$ is non-empty, for each $\bar{h} \in \mathcal{J}_{3}$, we define the function $\widetilde{F}_{\mathcal{J}, \bar{h}}: \Xi_{\mathcal{J}} \times$ $\Lambda_{\chi} \rightarrow \mathbb{R}^{\operatorname{dim} \Xi_{\mathcal{J}}+1}$ where

$$
\begin{aligned}
& \widetilde{F}_{\mathcal{J}, \bar{h}}(\xi, a, e):=\left(\widetilde{F}_{\mathcal{J}}(\xi, a, e), \widetilde{F}_{\mathcal{J}} \bar{h}^{4}(\xi, a, e)\right) \in \mathbb{R}^{\operatorname{dim} \Xi_{\mathcal{J}}+1} \\
& \text { where } \widetilde{F}_{\mathcal{J}}^{\bar{h} .4}(\xi, a, e):=\chi_{\bar{h}}\left(x_{\bar{h}}, x_{-\bar{h}}\right)+a_{\bar{h}}
\end{aligned}
$$

Note that $\widetilde{F}_{\mathcal{J}}$ and $\widetilde{F}_{\mathcal{J}, \bar{h}}$ are $C^{1}$ functions on their domains. Furthermore, for all $\mathcal{J} \in \mathcal{P}, \operatorname{dim} \Xi_{\mathcal{J}}=\operatorname{dim} \Xi$. The key lemma of this step is the following one.

Lemma 17 For every $\mathcal{J} \in \mathcal{P}$ such that $\mathcal{J}_{3} \neq \emptyset$ and for each $\bar{h} \in \mathcal{J}_{3}, 0$ is a regular value for $\widetilde{F}_{\mathcal{J}, \bar{h}}$.

Since the dimension $\operatorname{dim} \Xi_{\mathcal{J}}+1$ of the target space is strictly larger than the dimension of $\Xi_{\mathcal{J}}$, the Regular Value Theorem and a consequence of Sard's Theorem (see Theorems 24 and 26 in Appendix B) imply that for every $\mathcal{J} \in \mathcal{P}$ such that $\mathcal{J}_{3} \neq \emptyset$ there exists a full measure subset $\Omega_{\mathcal{J}, \bar{h}}$ of $\Lambda_{\chi}$ such that for each $(a, e) \in \Omega_{\mathcal{J}, \bar{h}}$,

$$
\left\{\xi \in \Xi_{\mathcal{J}}: \widetilde{F}_{\mathcal{J}, \bar{h}}(\xi, a, e)=0\right\}=\emptyset
$$

Now, let us consider an element $(a, e) \in \Phi(\widetilde{B})$. Then, there exist $\xi \in \Xi$ such that $\widetilde{F}(\xi, a, e)=0$ and $\bar{h} \in \mathcal{H}$ such that $\mu_{\bar{h}}=\chi_{\bar{h}}\left(x_{\bar{h}}, x_{-\bar{h}}\right)+a_{\bar{h}}=0$. Let $\mathcal{J} \in \mathcal{P}$ defined by

$\mathcal{J}_{1}:=\left\{h \in \mathcal{H}: \mu_{h}=0\right.$ and $\left.\chi_{h}\left(x_{h}, x_{-h}\right)+a_{h}>0\right\}$, $\mathcal{J}_{2}:=\left\{h \in \mathcal{H}: \mu_{h}>0\right.$ and $\left.\chi_{h}\left(x_{h}, x_{-h}\right)+a_{h}=0\right\}$, $\mathcal{J}_{3}:=\left\{h \in \mathcal{H}: \mu_{h}=\chi_{h}\left(x_{h}, x_{-h}\right)+a_{h}=0\right\}$.

One easily checks that $\widetilde{F}_{\mathcal{J}, \bar{h}}(\xi, a, e)=0$. So, $(a, e) \notin \Omega_{\mathcal{J}, \bar{h}}$. Hence, we have proven that $\Phi(\widetilde{B})$ is included in the finite union of the complements of $\Omega_{\mathcal{J}, \bar{h}}$ over all $\mathcal{J} \in \mathcal{P}$ such that $\mathcal{J}_{3} \neq \emptyset$ and $\bar{h} \in \mathcal{J}_{3}$. Since each of these sets is of measure zero, so too does $\Phi(\widetilde{B})$.

\subsection{Almost all perturbed economies are regular}

Take for fixed $\chi \in \mathcal{X}$. Observe that for given $(a, e) \in \Lambda_{\chi}$, by Definition 10, the economy $(\chi+a, e)$ is regular if $(a, e)$ belongs to the open and full measure set $\Lambda_{\chi}^{1}$ given by (9) in the previous subsection, and

$$
\forall \xi^{*} \in F_{\chi+a, e}^{-1}(0), \operatorname{rank} D_{\xi} F_{\chi+a, e}\left(\xi^{*}\right)=\operatorname{dim} \Xi
$$

From now on, with innocuous abuse of notation:

- the domain of $\widetilde{F}$ defined in (8) will be $\Xi \times \Lambda_{\chi}^{1}$ instead of $\Xi \times \Lambda_{\chi}$, 
- $\Phi$ denotes the restriction to $\widetilde{F}^{-1}(0)$ of the projection of $\Xi \times \Lambda_{\chi}^{1}$ onto $\Lambda_{\chi}^{1}$.

Importantly, one easily checks that from $(9)$, now $D_{\xi} \widetilde{F}(\xi, a, e)=D_{\xi} F_{\chi+a, e}(\xi)$ for every $(\xi, a, e) \in \widetilde{F}^{-1}(0)$. Then, from Assumptions 1 and $2, D_{\xi} \widetilde{F}$ is now a continuous function on $\widetilde{F}^{-1}(0)$.

Let us consider the following set

$$
\widetilde{C}:=\left\{(\xi, a, e) \in \widetilde{F}^{-1}(0): \operatorname{rank} D_{\xi} \widetilde{F}(\xi, a, e)<\operatorname{dim} \Xi\right\}
$$

We remark that

$$
\Lambda_{\chi}^{r}=\Lambda_{\chi}^{1} \backslash \Phi(\widetilde{C})
$$

Then, we have to prove that $\Phi(\widetilde{C})$ is closed in $\Lambda_{\chi}^{1}$ and $\Phi(\widetilde{C})$ is of measure zero.

Step 1. An element $(\xi, a, e)$ of $\widetilde{C}$ is characterized by the fact that the determinant of all the square submatrices of $D_{\xi} \widetilde{F}(\xi, a, e)$ of dimension $\operatorname{dim} \Xi$ is equal to zero. $\widetilde{C}$ is closed in $\widetilde{F}^{-1}(0)$ since the determinant is a continuous function and $D_{\xi} \widetilde{F}$ is continuous on $\widetilde{F}^{-1}(0)$. Then, $\Phi(\widetilde{C})$ is closed since $\Phi$ is proper ${ }^{14}$.

Step 2. We now show that $\Phi(\widetilde{C})$ is of measure zero in $\Lambda_{\chi}^{1}$. The key lemma is the following one.

Lemma 18 For every $\mathcal{J} \in \mathcal{P}$ such that $\mathcal{J}_{3}=\emptyset$, 0 is a regular value for $\widetilde{F}_{\mathcal{J}}$.

Then, from a consequence of Sard's Theorem (see Theorem 26 in Appendix B), for every $\mathcal{J} \in \mathcal{P}$ such that $\mathcal{J}_{3}=\emptyset$, there exists a full measure subset $\Omega_{\mathcal{J}}$ of $\Lambda_{\chi}^{1}$ such that for each $(a, e) \in \Omega_{\mathcal{J}}$ and for each $\xi^{*}$ such that $\widetilde{F}_{\mathcal{J}}\left(\xi^{*}, a, e\right)=0$, $\operatorname{rank} D_{\xi} \widetilde{F}_{\mathcal{J}}\left(\xi^{*}, a, e\right)=\operatorname{dim} \Xi_{\mathcal{J}}$.

Now, let us consider $(a, e) \in \Phi(\widetilde{C})$. Then, there exists $\xi \in \Xi$ such that $\widetilde{F}(\xi, a, e)=0$ and $\operatorname{rank} D_{\xi} \widetilde{F}(\xi, a, e)<\operatorname{dim} \Xi$. Let us consider the partition $\mathcal{J}$ associated to $(\xi, a, e)$ as in the previous subsection. Since $(a, e)$ belongs to $\Lambda_{\chi}^{1}$, then $\mathcal{J}_{3}=\emptyset$. Hence, one easily checks that $\widetilde{F}_{J}(\xi, a, e)=\widetilde{F}(\xi, a, e)$ on a neighborhood of $(\xi, a, e)$. So, the partial differential with respect to $\xi$ is the same and one concludes that $(a, e) \notin \Omega_{\mathcal{J}}$.

This prove that $\Phi(\widetilde{C})$ is included in the finite union of the complementary of $\Omega_{\mathcal{J}}$ over all $\mathcal{J} \in \mathcal{P}$ such that $\mathcal{J}_{3}=\emptyset$. Since these sets have zero measure, so too does $\Phi(\widetilde{C})$.

\footnotetext{
$\overline{14}$ The proof of the properness of $\Phi$ can be easily obtained using the same steps as in the proof of Lemma 16.
} 


\subsection{Generic regularity result in the space of economies}

In this subsection, we prove Corollary 13 . To show openess and density results, we follow a similar strategy to the one presented in Citanna, Kajii and Villanacci $(1998)^{15}$.

Let $\mathcal{R}$ be the set of economies $(\chi, e) \in \Theta$ such that $(\chi, e)$ is a regular economy. The density of $\mathcal{R}$ is a direct consequence of Theorem 12. Indeed, let $(\chi, e) \in \Theta$. Using Theorem 12, since an open and full measure subset is dense, one can find a sequence $\left(a^{\nu}, e^{\nu}\right)_{\nu \in \mathbb{N}} \subseteq \Lambda_{\chi}$ converging to $(0, e)$ such that $\left(\chi+a^{\nu}, e^{\nu}\right)$ is a regular economy for every $\nu \in \mathbb{N}$. From Remark 23 in Appendix B, the sequence $\left(\chi+a^{\nu}, e^{\nu}\right)_{\nu \in \mathbb{N}}$ converges to $(\chi, e)$, hence $\mathcal{R}$ is dense in $\Theta$.

We now show that $\mathcal{R}$ is open. We consider the global equilibrium function $F: \Xi \times \Theta \rightarrow \mathbb{R}^{\operatorname{dim} \Xi}$ defined by

$$
F(\xi, \chi, e):=F_{\chi, e}(\xi)
$$

By Assumptions 1 and 2 and Remark 22 in Appendix B, $F$ is continuous. Let us also define the restriction $\Pi$ to $F^{-1}(0)$ of the projection of $\Xi \times \Theta$ onto $\Theta$,

$$
\Pi:(\xi, \chi, e) \in F^{-1}(0) \rightarrow \Pi(\xi, \chi, e):=(\chi, e) \in \Theta
$$

The important property of $\Pi$ is given in the following lemma.

Lemma 19 The projection $\Pi: F^{-1}(0) \rightarrow \Theta$ is a proper function.

For every $h \in \mathcal{H}$, we define the following set

$$
B_{h}:=\left\{(\xi, a, e) \in F^{-1}(0): \mu_{h}=\chi_{h}\left(x_{h}, x_{-h}\right)=0\right\} \text { and } B:=\bigcup_{h \in \mathcal{H}} B_{h}
$$

Let

$$
\Theta^{1}:=\Theta \backslash \Pi(B)
$$

$\Theta^{1}$ is the set of $(\chi, e) \in \Theta$ such that for every $\xi^{*} \in F_{\chi, e}^{-1}(0), F_{\chi, e}$ is a $C^{1}$ function around $\xi^{*}$. Definition 10 implies that $\mathcal{R} \subseteq \Theta^{1}$.

$\Theta^{1}$ is open in $\Theta$. Indeed, $B$ is closed in $F^{-1}(0)$ as a consequence of Remark 22 (see Appendix $\mathrm{B}$ ), and $\Pi(B)$ is closed from the properness of $\Pi$.

Now, we prove that $\mathcal{R}$ is open in $\Theta^{1}$. We denote again by $F$ the mapping now defined on $\Xi \times \Theta^{1}$ instead of $\Xi \times \Theta$ and by $\Pi$ the restriction to $F^{-1}(0)$ of the projection from $\Xi \times \Theta^{1}$ onto $\Theta^{1}$. Importantly, one easily checks that from (14), now $D_{\xi} F(\xi, \chi, e)=D_{\xi} F_{\chi, e}(\xi)$ for every $(\xi, a, e) \in F^{-1}(0)$. Then, from

\footnotetext{
$\overline{15}$ Observe that in Citanna, Kajii and Villanacci (1998), openess and density results mainly concern constrained suboptimality issues.
} 
Assumptions 1 and 2 and Remark 22 in Appendix B, $D_{\xi} F$ is now a continuous function on $F^{-1}(0)$.

Let us consider the set

$$
C:=\left\{(\xi, \chi, e) \in F^{-1}(0): \operatorname{rank} D_{\xi} F(\xi, \chi, e)<\operatorname{dim} \Xi\right\}
$$

Definition 10 implies that

$$
\mathcal{R}=\Theta^{1} \backslash \Pi(C)
$$

$C$ is closed due to the continuity of the determinant function and of $D_{\xi} F$, $\Pi(C)$ is closed due to the properness of $\Pi$. Consequently, $\mathcal{R}$ is open in $\Theta^{1}$.

\section{Appendix A}

We start by a selection property of the consumption sets, which will play a fundamental role in the properness result used to show openess properties in the space of economies $\Theta$ (see the proof of Lemma 19). Next, we show all the lemmas stated in Section 7.

Proposition 20 Let $h \in \mathcal{H}, \Theta_{h}$ is the projection of $\Theta$ onto $C^{0-2}(T, \mathbb{R}) \times \mathbb{R}_{++}^{C}$ equipped with the metric induced by the one on $C^{0-2}(T, \mathbb{R}) \times \mathbb{R}^{C 16}$. For each $h \in \mathcal{H}$, there is a continuous function $\widehat{x}_{h}: \mathbb{R}_{+}^{C(H-1)} \times \Theta_{h} \rightarrow \mathbb{R}_{++}^{C}$ such that for each $\left(z, \chi_{h}, e_{h}\right) \in \mathbb{R}_{+}^{C(H-1)} \times \Theta_{h}, \chi_{h}\left(\widehat{x}_{h}\left(z, \chi_{h}, e_{h}\right), z\right)>0$ and $\widehat{x}_{h}\left(z, \chi_{h}, e_{h}\right) \ll e_{h}$.

Proof of Proposition 20. First, observe that $\mathbb{R}_{+}^{C(H-1)} \times \Theta_{h}$ is a metric space. Second, the correspondence $\phi_{h}: \mathbb{R}_{+}^{C(H-1)} \times \Theta_{h} \rightrightarrows \mathbb{R}^{C}$ defined by $\phi_{h}\left(z, \chi_{h}, e_{h}\right):=\left\{x_{h} \in \mathbb{R}_{++}^{C}: \chi_{h}\left(x_{h}, z\right)>0\right.$ and $\left.x_{h} \ll e_{h}\right\}$ is non-empty convex valued by (1) and Definition 11, and by Point 2 of Assumption 2. We now prove that $\phi_{h}$ has open fiber, that is, for all $x_{h} \in \mathbb{R}_{+}^{C}$, the following set

$$
\phi_{h}^{-1}\left(x_{h}\right):=\left\{\left(z, \chi_{h}, e_{h}\right) \in \mathbb{R}_{+}^{C(H-1)} \times \Theta_{h}: \chi_{h}\left(x_{h}, z\right)>0 \text { and } x_{h} \ll e_{h}\right\}
$$

is open in $\mathbb{R}_{+}^{C(H-1)} \times \Theta_{h}$. This follows from Remark 22 in Appendix B, which shows that the application $\left(\chi_{h}, x_{h}, z\right) \rightarrow \chi_{h}\left(x_{h}, z\right)$ is continuous on $C^{0-2}(T, \mathbb{R}) \times$ $T$. Finally, we get the desired result since the correspondence $\phi_{h}$ satisfies the assumptions of Michael's Selection Theorem (see Florenzano, 2003).

Proof of Lemma 16. The proof is a direct consequence of Lemma 19 since the mapping $(\chi, a) \rightarrow \chi+a$ is continuous on $\left(C^{0-2}(T, \mathbb{R})\right)^{H} \times \mathbb{R}^{H}$ (see Remark 23 in Appendix B).

${ }^{16}$ The metric on $C^{0-2}(T, \mathbb{R}) \times \mathbb{R}^{C}$ is given by summing the metric $\widetilde{d}$ on $C^{0-2}(T, \mathbb{R})$ (see Definition 21 in Appendix B) and the Euclidean metric on $\mathbb{R}^{C}$. 
Proof of Lemma 17. The function $\widetilde{F}_{\mathcal{J}, \bar{h}}$ is defined in (12). We have to show that for each $\left(\xi^{*}, a^{*}, e^{*}\right) \in \widetilde{F}_{\mathcal{J}, \bar{h}}^{-1}(0)$, the Jacobian matrix $D_{\xi, a, e} \widetilde{F}_{\mathcal{J}, \bar{h}}\left(\xi^{*}, a^{*}, e^{*}\right)$ has full row rank.

Let $\Delta:=\left(\left(\Delta x_{h}, \Delta \lambda_{h}, \Delta \mu_{h}\right)_{h \in \mathcal{H}}, \Delta p \backslash, \Delta w\right) \in \mathbb{R}^{(C+2) H} \times \mathbb{R}^{C-1} \times \mathbb{R}$. It is enough to show that $\Delta D_{\xi, a, e} \widetilde{F}_{\mathcal{J}, \bar{h}}\left(\xi^{*}, a^{*}, e^{*}\right)=0$ implies $\Delta=0$. To prove it, we consider the computation of the partial Jacobian matrix with respect to the following variables

$$
\left(\left(x_{h}, \lambda_{h}, e_{h}\right)_{h \in \mathcal{H}},\left(a_{h^{\prime}}\right)_{h^{\prime} \in \mathcal{J}_{2}}, a_{\bar{h}}, p^{\backslash}\right)
$$

The partial system $\Delta D_{\xi, a, e} \widetilde{F}_{\mathcal{J}, \bar{h}}\left(\xi^{*}, a^{*}, e^{*}\right)=0$ is written in detail below.

$$
\left\{\begin{array}{l}
\sum_{h \in \mathcal{H}} \Delta x_{h} D_{x_{k} x_{h}}^{2} u_{h}\left(x_{h}^{*}, x_{-h}^{*}\right)+\sum_{h^{\prime} \in \mathcal{J}_{2}} \mu_{h^{\prime}}^{*} \Delta x_{h^{\prime}} D_{x_{k} x_{h^{\prime}}}^{2} \chi_{h^{\prime}}\left(x_{h^{\prime}}^{*}, x_{-h^{\prime}}^{*}\right)+ \\
\sum_{h^{\prime} \in \mathcal{J}_{2}} \Delta \mu_{h^{\prime}} D_{x_{k}} \chi_{h^{\prime}}\left(x_{h^{\prime}}^{*}, x_{-h^{\prime}}^{*}\right)-\Delta \lambda_{k} p^{*}+\Delta p \backslash\left[I_{C-1} \mid 0\right]+ \\
\Delta w D_{x_{k}} \chi_{\bar{h}}\left(x_{\bar{h}}^{*}, x_{-\bar{h}}^{*}\right)=0, \forall k \in \mathcal{H} \\
-\Delta x_{h} \cdot p^{*}=0, \forall h \in \mathcal{H} \\
\Delta x_{h} \cdot D_{x_{h}} \chi_{h}\left(x_{h}^{*}, x_{-h}^{*}\right)+\Delta \mu_{h}=0, \forall h \in \mathcal{J}_{1} \cup \mathcal{J}_{3} \\
\Delta x_{h^{\prime}} \cdot D_{x_{h^{\prime}}} \chi_{h^{\prime}}\left(x_{h^{\prime}}^{*}, x_{-h^{\prime}}^{*}\right)=0, \forall h^{\prime} \in \mathcal{J}_{2} \\
\Delta \lambda_{h} p^{*}-\Delta p \backslash\left[I_{C-1} \mid 0\right]=0, \forall h \in \mathcal{H} \\
\Delta \mu_{h^{\prime}}=0, \forall h^{\prime} \in \mathcal{J}_{2} \\
\Delta w=0 \\
\sum_{h \in \mathcal{H}} \lambda_{h}^{*} \Delta x_{h}^{\backslash}+\sum_{h \in \mathcal{H}} \Delta \lambda_{h}\left(x_{h}^{* \backslash}-e_{h}^{* \backslash}\right)=0
\end{array}\right.
$$

Since $p^{* C}=1$, we get $\Delta \lambda_{h}=0$ for each $h \in \mathcal{H}$ and $\Delta p \backslash=0$. Then, the relevant equations of the above system become

$$
\left\{\begin{array}{l}
\forall k \in \mathcal{H}, \\
\sum_{h \in \mathcal{H}} \Delta x_{h} D_{x_{k} x_{h}}^{2} u_{h}\left(x_{h}^{*}, x_{-h}^{*}\right)+\sum_{h^{\prime} \in \mathcal{J}_{2}} \mu_{h^{\prime}}^{*} \Delta x_{h^{\prime}} D_{x_{k} x_{h^{\prime}}}^{2} \chi_{h^{\prime}}\left(x_{h^{\prime}}^{*}, x_{-h^{\prime}}^{*}\right)=0 \\
-\Delta x_{h} \cdot p^{*}=0, \forall h \in \mathcal{H} \\
\Delta x_{h} \cdot D_{x_{h}} \chi_{h}\left(x_{h}^{*}, x_{-h}^{*}\right)+\Delta \mu_{h}=0, \forall h \in \mathcal{J}_{1} \cup \mathcal{J}_{3} \\
\Delta x_{h^{\prime}} \cdot D_{x_{h^{\prime}}} \chi_{h^{\prime}}\left(x_{h^{\prime}}^{*}, x_{-h^{\prime}}^{*}\right)=0, \forall h^{\prime} \in \mathcal{J}_{2} \\
\sum_{h \in \mathcal{H}} \lambda_{h}^{*} \Delta x_{h}=0
\end{array}\right.
$$


Observe that from $\widetilde{F}_{\mathcal{J}}^{h .1}\left(\xi^{*}, a^{*}, e^{*}\right)=0$ and the above system, we get

$$
\left(\Delta x_{h}\right)_{h \in \mathcal{H}} \in \prod_{h \in \mathcal{H}} \operatorname{Ker} D_{x_{h}} u_{h}\left(x_{h}^{*}, x_{-h}^{*}\right)
$$

Indeed, $D_{x_{h}} u_{h}\left(x_{h}^{*}, x_{-h}^{*}\right) \cdot \Delta x_{h}=\lambda_{h}^{*} p^{*} \cdot \Delta x_{h}=0$ for each $h \in \mathcal{J}_{1} \cup \mathcal{J}_{3}$, and $D_{x_{h^{\prime}}} u_{h^{\prime}}\left(x_{h^{\prime}}^{*}, x_{-h^{\prime}}^{*}\right) \cdot \Delta x_{h^{\prime}}=\lambda_{h^{\prime}}^{*} p^{*} \cdot \Delta x_{h^{\prime}}-\mu_{h^{\prime}}^{*} D_{x_{h^{\prime}}} \chi_{h^{\prime}}\left(x_{h^{\prime}}^{*}, x_{-h^{\prime}}^{*}, e_{h^{\prime}}^{*}\right) \cdot \Delta x_{h^{\prime}}=0$ for each $h^{\prime} \in \mathcal{J}_{2}$. Now, for each $h \in \mathcal{H}$ define

$$
v_{h}:=\lambda_{h}^{*} \Delta x_{h}
$$

From equations in (16) and the above conditions, it follows that the vector $\left(x_{h}^{*}, v_{h}\right)_{h \in \mathcal{H}} \in \mathbb{R}_{++}^{C H} \times \mathbb{R}^{C H}$ satisfies the following conditions

$$
\sum_{h \in \mathcal{H}} v_{h}=0 \text { and }\left(v_{h}\right)_{h \in \mathcal{H}} \in \prod_{h \in \mathcal{H}} \operatorname{Ker} D_{x_{h}} u_{h}\left(x_{h}^{*}, x_{-h}^{*}\right)
$$

and

$$
v_{h^{\prime}} \in \operatorname{Ker} D_{x_{h^{\prime}}} \chi_{h^{\prime}}\left(x_{h^{\prime}}^{*}, x_{-h^{\prime}}^{*}\right) \text { for each } h^{\prime} \in \mathcal{J}_{2}
$$

Now, observe that the first equation of system (16) implies that for each $k \in \mathcal{H}$

$$
\sum_{h \in \mathcal{H}} \Delta x_{h} D_{x_{k} x_{h}}^{2} u_{h}\left(x_{h}^{*}, x_{-h}^{*}\right)\left(v_{k}\right)=-\sum_{h^{\prime} \in \mathcal{J}_{2}} \mu_{h^{\prime}}^{*} \Delta x_{h^{\prime}} D_{x_{k} x_{h^{\prime}}}^{2} \chi_{h^{\prime}}\left(x_{h^{\prime}}^{*}, x_{-h^{\prime}}^{*}\right)\left(v_{k}\right)
$$

Since $\lambda_{h}^{*} \neq 0$ for all $h \in \mathcal{H}$, then it follows by (17) that for each $k \in \mathcal{H}$

$$
\sum_{h \in \mathcal{H}} \frac{v_{h}}{\lambda_{h}^{*}} D_{x_{k} x_{h}}^{2} u_{h}\left(x_{h}^{*}, x_{-h}^{*}\right)\left(v_{k}\right)=-\sum_{h^{\prime} \in \mathcal{H}_{2}} \mu_{h^{\prime}}^{*} \frac{v_{h^{\prime}}}{\lambda_{h^{\prime}}^{*}} D_{x_{k} x_{h^{\prime}}}^{2} \chi_{h^{\prime}}\left(x_{h^{\prime}}^{*}, x_{-h^{\prime}}^{*}\right)\left(v_{k}\right)
$$

Summing up $k \in \mathcal{H}$, we get

$$
\sum_{h \in \mathcal{H}} \frac{v_{h}}{\lambda_{h}^{*}} \sum_{k \in \mathcal{H}} D_{x_{k} x_{h}}^{2} u_{h}\left(x_{h}^{*}, x_{-h}^{*}\right)\left(v_{k}\right)=-\sum_{h^{\prime} \in \mathcal{J}_{2}} \frac{\mu_{h^{\prime}}^{*}}{\lambda_{h^{\prime}}^{*}} v_{h^{\prime}} \sum_{k \in \mathcal{H}} D_{x_{k} x_{h^{\prime}}}^{2} \chi_{h^{\prime}}\left(x_{h^{\prime}}^{*}, x_{-h^{\prime}}^{*}\right)\left(v_{k}\right)
$$

Since $\lambda_{h^{\prime}}^{*}>0$ and $\mu_{h^{\prime}}^{*}>0$ for each $h^{\prime} \in \mathcal{J}_{2}$, then from the above condition, (18) and Point 2 of Assumption 9 we have that

$$
\sum_{h \in \mathcal{H}} \frac{1}{\lambda_{h}^{*}} v_{h} \sum_{k \in \mathcal{H}} D_{x_{k} x_{h}}^{2} u_{h}\left(x_{h}^{*}, x_{-h}^{*}\right)\left(v_{k}\right) \geq 0
$$

Therefore, since $\lambda_{h}^{*}>0$ for all $h \in \mathcal{H}$, Point 1 of Assumption 9 implies that $v_{h}=0$ for each $h \in \mathcal{H}$. By (17), we get $\Delta x_{h}=0$ for all $h \in \mathcal{H}$. Then, by system (16), we have that $\Delta \mu_{h}=0$ for each $h \in \mathcal{J}_{1} \cup \mathcal{J}_{3}$. Thus, $\Delta=0$.

Proof of Lemma 18. The function $\widetilde{F}_{\mathcal{J}}$ is defined in (8). We have to show that for each $\left(\xi^{*}, a^{*}, e^{*}\right) \in \widetilde{F}_{\mathcal{J}}^{-1}(0)$, the Jacobian matrix $D_{\xi, a, e} \widetilde{F}_{\mathcal{J}}\left(\xi^{*}, a^{*}, e^{*}\right)$ has full row rank. 
Let $\Delta:=\left(\left(\Delta x_{h}, \Delta \lambda_{h}, \Delta \mu_{h}\right)_{h \in \mathcal{H}}, \Delta p^{\prime}\right) \in \mathbb{R}^{(C+2) H} \times \mathbb{R}^{C-1}$. It is enough to show that $\Delta D_{\xi, a, e} \widetilde{F}_{\mathcal{J}}\left(\xi^{*}, a^{*}, e^{*}\right)=0$ implies $\Delta=0$. To prove it, we consider the computation of the partial Jacobian matrix with respect to the following variables

$$
\left(\left(x_{h}, \lambda_{h}, e_{h}\right)_{h \in \mathcal{H}},\left(a_{h^{\prime}}\right)_{h^{\prime} \in \mathcal{J}_{2}}, p^{\backslash}\right)
$$

and the corresponding partial system. Then, the proof follows the same steps as in the proof of Lemma 17. Indeed, note that there is a slight difference between this partial system and the one given in (15): now $\mathcal{J}_{3}=\emptyset$. Then, we have one variable less, i.e., $\Delta w$, and one equation less, i.e., $\Delta w=0$.

Proof of Lemma 19. We show that any sequence $\left(\xi^{\nu}, \chi^{\nu}, e^{\nu}\right)_{\nu \in \mathbb{N}} \subseteq F^{-1}(0)$, up to a subsequence, converges to an element of $F^{-1}(0)$, knowing that $\left(\chi^{\nu}, e^{\nu}\right)_{\nu \in \mathbb{N}} \subseteq$ $\Theta$ converges to $\left(\chi^{*}, e^{*}\right) \in \Theta$.

We recall that $\xi^{\nu}=\left(x^{\nu}, \lambda^{\nu}, \mu^{\nu}, p^{\nu \backslash}\right)$.

- $\left(x^{\nu}\right)_{v \in \mathbb{N}}$, up to a subsequence, converges to $x^{*} \in \mathbb{R}_{++}^{C H}$.

$\left(x^{\nu}\right)_{v \in \mathbb{N}} \subseteq \mathbb{R}_{++}^{C H}$. From $F^{M}\left(\xi^{\nu}, \chi^{\nu}, e^{\nu}\right)=0$ and $F^{k .2}\left(\xi^{\nu}, \chi^{\nu}, e^{\nu}\right)=0, x_{k}^{\nu}=$ $\sum_{h \in \mathcal{H}} e_{h}^{\nu}-\sum_{h \neq k} x_{h}^{\nu} \leq \sum_{h \in \mathcal{H}} e_{h}^{\nu}$ for each $k \in \mathcal{H}$. Then, $\left(x^{\nu}\right)_{\nu \in \mathbb{N}}$ is bounded from above by an element of $\mathbb{R}_{++}^{C H}$, since for each $h \in \mathcal{H},\left(e_{h}^{\nu}\right)_{\nu \in \mathbb{N}}$ converges to $e_{h}^{*} \in E_{\chi_{h}^{*}} \subseteq \mathbb{R}_{++}^{C}$. Then, $\left(x^{\nu}\right)_{\nu \in \mathbb{N}}$, up to a subsequence, converges to $x^{*} \geq 0$.

Now, we prove that $x_{h}^{*} \gg 0$ for each $h \in \mathcal{H}$.

By $F^{h .1}\left(\xi^{\nu}, \chi^{\nu}, e^{\nu}\right)=0, F^{h .2}\left(\xi^{\nu}, \chi^{\nu}, e^{\nu}\right)=0$ and $F^{h .3}\left(\xi^{\nu}, \chi^{\nu}, e^{\nu}\right)=0$, it follows that $u_{h}\left(x_{h}^{\nu}, x_{-h}^{\nu}\right) \geq u_{h}\left(\hat{x}_{h}\left(x_{-h}^{\nu}, \chi_{h}^{\nu}, e_{h}^{\nu}\right), x_{-h}^{\nu}\right)$ for every $\nu \in \mathbb{N}$, where $\hat{x}_{h}$ is the continuous selection function given by Proposition 20. Define $\mathbf{1}:=$ $(1, \ldots, 1) \in \mathbb{R}_{++}^{C}$, from Point 2 of Assumption 1 we have that for each $\varepsilon>0$, $u_{h}\left(x_{h}^{\nu}+\varepsilon \mathbf{1}, x_{-h}^{\nu}\right) \geq u_{h}\left(\hat{x}_{h}\left(x_{-h}^{\nu}, \chi_{h}^{v}, e^{\nu}\right), x_{-h}^{\nu}\right)$ for every $\nu \in \mathbb{N}$. So taking the limit on $\nu$, since $\left(\chi_{h}^{\nu}, e_{h}^{\nu}\right)_{\nu \in \mathbb{N}}$ converges to $\left(\chi_{h}^{*}, e_{h}^{*}\right) \in \Theta_{h}$, and $u_{h}$ and $\hat{x}_{h}$ are continuous functions (see Point 1 of Assumption 1 and Proposition 20), then $u_{h}\left(x_{h}^{*}+\varepsilon \mathbf{1}, x_{-h}^{*}\right) \geq u_{h}\left(\hat{x}_{h}\left(x_{-h}^{*}, \chi_{h}^{*}, e_{h}^{*}\right), x_{-h}^{*}\right):=\underline{u}_{h}$ for each $\varepsilon>0$. By Point 4 of Assumption $1, x_{h}^{*} \in \mathbb{R}_{++}^{C}$ since $x_{h}^{*}$ belongs to the set $c l_{\mathbb{R}^{C}}\left\{x_{h} \in \mathbb{R}_{++}^{C}\right.$ : $\left.u_{h}\left(x_{h}, x_{-h}^{*}\right) \geq \underline{u}_{h}\right\}$.

- $\left(\lambda^{\nu}, \mu^{\nu}\right)_{v \in \mathbb{N}}$, up to a subsequence, converges to $\left(\lambda^{*}, \mu^{*}\right) \in \mathbb{R}_{+}^{H} \times \mathbb{R}_{+}^{H}$.

It is enough to show that $\left(\lambda_{h}^{\nu} p^{\nu}, \mu_{h}^{\nu}\right)_{\nu \in \mathbb{N}}$ is bounded for each $h \in \mathcal{H}$. Then, $\left(\lambda_{h}^{\nu} p^{\nu}, \mu_{h}^{\nu}\right)_{\nu \in \mathbb{N}} \subseteq \mathbb{R}_{++}^{C} \times \mathbb{R}_{+}$, up to a subsequence, converges to $\left(\pi_{h}^{*}, \mu_{h}^{*}\right) \in$ $\mathbb{R}_{+}^{C} \times \mathbb{R}_{+}$, and $\lambda_{h}^{*}=\pi_{h}^{* C}$ since $p^{v C}=1$ for each $\nu \in \mathbb{N}$.

Suppose otherwise that there is a subsequence of $\left(\lambda_{h}^{\nu} p^{\nu}, \mu_{h}^{\nu}\right)_{\nu \in \mathbb{N}}$ (that without loss of generality we continue to denote with $\left.\left(\lambda_{h}^{\nu} p^{\nu}, \mu_{h}^{\nu}\right)_{v \in \mathbb{N}}\right)$ such that 
$\left\|\left(\lambda_{h}^{\nu} p^{\nu}, \mu_{h}^{\nu}\right)\right\| \rightarrow+\infty$. Consider the sequence $\left(\frac{\left(\lambda_{h}^{\nu} p^{\nu}, \mu_{h}^{\nu}\right)}{\left\|\left(\lambda_{h}^{\nu} p^{\nu}, \mu_{h}^{\nu}\right)\right\|}\right)_{\nu \in \mathbb{N}}$ in the sphere, a compact set. Then, up to a subsequence $\left(\frac{\left(\lambda_{h}^{\nu} p^{\nu}, \mu_{h}^{\nu}\right)}{\left\|\left(\lambda_{h}^{\nu} p^{\nu}, \mu_{h}^{\nu}\right)\right\|}\right) \rightarrow\left(\pi_{h}, \mu_{h}\right) \neq 0 . \pi_{h} \geq 0$ and $\mu_{h} \geq 0$, since $\lambda_{h}^{\nu} p^{\nu} \gg 0$ and $\mu_{h}^{\nu} \geq 0$ for each $\nu \in \mathbb{N}$. By $F^{h .1}\left(\xi^{\nu}, \chi^{\nu}, e^{\nu}\right)=0$ for each $\nu \in \mathbb{N}$, we get $\lambda_{h}^{\nu} p^{\nu}=D_{x_{h}} u_{h}\left(x_{h}^{\nu}, x_{-h}^{\nu}\right)+\mu_{h}^{\nu} D_{x_{h}} \chi_{h}^{\nu}\left(x_{h}^{\nu}, x_{-h}^{\nu}\right)$. Now, divide both sides by $\left\|\left(\lambda_{h}^{\nu} p^{\nu}, \mu_{h}^{\nu}\right)\right\|$ and take the limits. From Point 1 of Assumption 1 and Remark 22, we get

$$
\pi_{h}=\mu_{h} D_{x_{h}} \chi_{h}^{*}\left(x_{h}^{*}, x_{-h}^{*}\right)
$$

Then, $\mu_{h}>0$. Otherwise we get $\left(\pi_{h}, \mu_{h}\right)=0$. From Point 4 of Assumption 2, we have that $D_{x_{h}} \chi_{h}^{*}\left(x_{h}^{*}, x_{-h}^{*}\right) \neq 0$. Then, $\pi_{h} \neq 0$. From Kuhn-Tucker necessary and sufficient conditions, we have that

$$
\begin{aligned}
\pi_{h} \cdot x_{h}^{*}= & \min _{x_{h} \in \mathbb{R}_{++}^{C}} \pi_{h} \cdot x_{h} \\
& \text { subject to } \chi_{h}^{*}\left(x_{h}, x_{-h}^{*}\right) \geq 0
\end{aligned}
$$

By $F^{h .2}\left(\xi^{\nu}, \chi^{\nu}, e^{\nu}\right)=0$, we get $\lambda_{h}^{\nu} p^{\nu} \cdot x_{h}^{\nu}=\lambda_{h}^{\nu} p^{\nu} \cdot e_{h}^{\nu}$ for each $\nu \in \mathbb{N}$. Now, divide both sides by $\left\|\left(\lambda_{h}^{\nu} p^{\nu}, \mu_{h}^{\nu}\right)\right\|$ and take the limits. We get $\pi_{h} \cdot x_{h}^{*}=\pi_{h} \cdot e_{h}^{*}$. By (1), there is $\widetilde{x}_{h} \in \mathbb{R}_{++}^{C}$ such that $\chi_{h}^{*}\left(\widetilde{x}_{h}, x_{-h}^{*}\right)>0$ and $\pi_{h} \cdot \widetilde{x}_{h}<\pi_{h} \cdot e_{h}^{*}=\pi_{h} \cdot x_{h}^{*}$ which contradict (19).

- $\left(p^{\nu \backslash}\right)_{\nu \in \mathbb{N}}$, up to a subsequence, converges to $p^{* \backslash} \in \mathbb{R}_{++}^{C-1}$.

Taking the limit, from Remark 22, Points 1 and 2 of Assumption 1, and Points 1 and 5 of Assumption 2, we get $\lambda_{k}^{*}=D_{x_{k}^{C}} u_{k}\left(x_{k}^{*}, x_{-k}^{*}\right)+\mu_{k}^{*} D_{x_{k}^{C}} \chi_{k}^{*}\left(x_{k}^{*}, x_{-k}^{*}\right)>$ 0 for some $k=h(C) \in \mathcal{H}$. From the previous step, $\left(\lambda_{k}^{\nu} p^{v \backslash}\right)_{\nu \in \mathbb{N}}$ admits a subsequence converging to $\pi_{k}^{* \backslash} \geq 0$. Then, $\left(p^{v \backslash}\right)_{\nu \in \mathbb{N}}$, up to a subsequence, converges to $p^{*} \geq 0$, since $\lambda_{k}^{*}>0$. Now, suppose that there is $c \neq C$, such that $p^{* c}=0$. Taking the limit, from Remark 22, Points 1 and 2 of Assumption 1 , and Points 1 and 5 of Assumption 2, for some $k^{\prime}=h(c) \in \mathcal{H}$ we get $0<$ $D_{x_{k^{\prime}}^{c}} u_{k^{\prime}}\left(x_{k^{\prime}}^{*}, x_{-k^{\prime}}^{*}\right)+\mu_{k^{\prime}}^{*} D_{x_{k^{\prime}}^{c}} \chi_{k^{\prime}}^{*}\left(x_{k^{\prime}}^{*}, x_{-k^{\prime}}^{*}\right)=\lambda_{k^{\prime}}^{*} p^{* c}=0$, which is a contradiction.

- $\lambda^{*} \in \mathbb{R}_{++}^{H}$.

Otherwise, suppose that $\lambda_{h}^{*}=0$ for some $h \in \mathcal{H}$. By $F^{h .1}\left(\xi^{\nu}, \chi^{\nu}, e^{\nu}\right)=0$, we get $\lambda_{h}^{\nu} p^{\nu}=D_{x_{h}} u_{h}\left(x_{h}^{\nu}, x_{-h}^{\nu}\right)+\mu_{h}^{\nu} D_{x_{h}} \chi_{h}^{\nu}\left(x_{h}^{\nu}, x_{-h}^{\nu}\right)$ for each $\nu \in \mathbb{N}$. Taking the limit, from Remark 22 and Point 1 of Assumption 1, we get $0=\lambda_{h}^{*} p^{*}=$ $D_{x_{h}} u_{h}\left(x_{h}^{*}, x_{-h}^{*}\right)+\mu_{h}^{*} D_{x_{h}} \chi_{h}^{*}\left(x_{h}^{*}, x_{-h}^{*}\right)$. By Point 2 of Assumption 1 and Point 4 of Assumption 2, we get $0<D_{x_{h}^{c}} u_{h}\left(x_{h}^{*}, x_{-h}^{*}\right)+\mu_{h}^{*} D_{x_{h}^{c}} \chi_{h}^{*}\left(x_{h}^{*}, x_{-h}^{*}\right)=\lambda_{h}^{*} p^{* c}=0$ for some good $c$, which is a contradiction.

\section{Appendix B}




\section{Topology of the $C^{0-2}$ uniform convergence on compacta}

Let $T:=\mathbb{R}_{++}^{C} \times \mathbb{R}_{+}^{C(H-1)}$. We are interested on continuous functions defined on $T$ which are $C^{2}$ in the interior of $T$ (see Point 1 of Assumption 2). Then, first, define the following set

$$
C^{0-2}(T, \mathbb{R}):=\left\{f \in C^{0}(T, \mathbb{R}): f_{\mid \operatorname{Int} T} \in C^{2}(\operatorname{Int} T, \mathbb{R})\right\}
$$

where Int $T$ denotes the interior of $T$ and $f_{\mid \operatorname{Int} T}$ denotes the restriction of $f$ to Int $T$. The topology on $C^{0-2}(T, \mathbb{R})$ of the $C^{0-2}$ uniform convergence on compacta is a "combination" of the topology on $C^{0}(T, \mathbb{R})$ of the $C^{0}$ uniform convergence on compacta and of the topology on $C^{2}(\operatorname{Int} T, \mathbb{R})$ of the $C^{2}$ uniform convergence on compacta.

Definition 21 The topology on $C^{0-2}(T, \mathbb{R})$ of the $C^{0-2}$ uniform convergence on compacta is the topology generated by the metric $\widetilde{d}$ defined by

$$
\forall(f, g) \in C^{0-2}(T, \mathbb{R}), \widetilde{d}(f, g):=d_{0}(f, g)+d_{2}\left(f_{\mid \operatorname{Int} T}, g_{\mid \operatorname{Int} T}\right)
$$

where $d_{2}$ is the metric d given in Allen (1981), p. 281, and $d_{0}$ is defined in an analogous way: let $\left\{T_{n}\right\}$ be a sequence of compact subsets of $T$ such that $\bigcup_{n=1}^{\infty} T_{n}=T, d_{0}(f, g):=\sum_{n=1}^{\infty} \frac{1}{2^{n}} \min \left\{\|f-g\|_{0, T_{n}}, 1\right\}$ for $f$ and $g$ in $C^{0}(T, \mathbb{R})$, where $\|\cdot\|_{0, T_{n}}$ is defined by $\|w\|_{0, T_{n}}:=\sup _{x \in T_{n}}|w(x)|$ for $w \in C^{0}\left(T_{n}, \mathbb{R}\right)$.

Remark 22 Observe that, by definition $f_{n} \stackrel{\widetilde{d}}{\rightarrow} \bar{f}$ in $C^{0-2}(T, \mathbb{R})$ if and only if

$$
f_{n} \stackrel{d_{0}}{\rightarrow} \bar{f} \text { in } C^{0}(T, \mathbb{R}) \text { and } f_{n \mid \operatorname{Int} T} \stackrel{d_{2}}{\rightarrow} \bar{f}_{\mid \operatorname{Int} T} \text { in } C^{2}(\operatorname{Int} T, \mathbb{R})
$$

That is, $f_{n} \stackrel{\widetilde{d}}{\rightarrow} \bar{f}$ in $C^{0-2}(T, \mathbb{R})$ if and only if $\left(f_{n}\right)_{n \in \mathbb{N}}$ converges uniformly to $\bar{f}$ on any compact set included in $T$, and $\left(f_{n \mid \operatorname{Int} T}\right)_{n \in \mathbb{N}},\left(D f_{n \mid \operatorname{Int} T}\right)_{n \in \mathbb{N}}$ and $\left(D^{2} f_{n \mid \operatorname{Int} T}\right)_{n \in \mathbb{N}}$ converge uniformly to $\bar{f}_{\mid \operatorname{Int} T}, D \bar{f}_{\mid \operatorname{Int} T}$ and $D^{2} \bar{f}_{\mid \operatorname{Int} T}$ respectively, on any compact set included in $\operatorname{Int} T$.

Consequentially, the mapping $(f, x) \rightarrow f(x)$ is continuous on $C^{0-2}(T, \mathbb{R}) \times T$ if and only if

(1) the mapping $(f, x) \rightarrow f(x)$ is continuous on $C^{0}(T, \mathbb{R}) \times T$, and

(2) the mappings $\left(f_{\mid \operatorname{Int} T}, x\right) \rightarrow f_{\mid \operatorname{Int} T}(x),\left(D f_{\mid \operatorname{Int} T}, x\right) \rightarrow D f_{\mid \operatorname{Int} T}(x)$, and $\left(D^{2} f_{\mid \operatorname{Int} T}, x\right) \rightarrow D^{2} f_{\mid \operatorname{Int} T}(x)$ are continuous on $C^{2}(\operatorname{Int} T, \mathbb{R}) \times \operatorname{Int} T$.

Since (1) holds true by definition of topology of the $C^{0}$ uniform convergence on compacta and (2) holds true by definition of topology of the $C^{2}$ uniform convergence on compacta, then the mapping $(f, x) \rightarrow f(x)$ is continuous on $C^{0-2}(T, \mathbb{R}) \times T$. 
Remark 23 One easily checks that the mapping $(f, a) \rightarrow f+a$ is continuous on $C^{0-2}(T, \mathbb{R}) \times \mathbb{R}$.

Finally, we remark that the topology of the $C^{0}$ uniform convergence on any compact set included in $T$ is uniquely used to show Proposition 20 which plays a fundamental role in the proof of Lemma 19.

\section{Regular values and transversality}

The theory of general economic equilibrium from a differentiable prospective is based on results from differential topology. Following are the ones used in our analysis. These results, as well as generalizations on these issues, can be found for instance in Guillemin and Pollack (1974), Hirsch (1976), Mas-Colell (1985) and Villanacci et al. (2002).

Theorem 24 (Regular Value Theorem) Let $M, N$ be $C^{r}$ manifolds of dimensions $m$ and $n$, respectively. Let $f: M \rightarrow N$ be a $C^{r}$ function. Assume $r>\max \{m-n, 0\}$. If $y \in N$ is a regular value for $f$, then

(1) if $m<n, f^{-1}(y)=\emptyset$,

(2) if $m \geq n$, either $f^{-1}(y)=\emptyset$, or $f^{-1}(y)$ is an $(m-n)$-dimensional submanifold of $M$.

Corollary 25 Let $M, N$ be $C^{r}$ manifolds of the same dimension. Let $f$ : $M \rightarrow N$ be a $C^{r}$ function. Assume $r \geq 1$. Let $y \in N$ a regular value for $f$ such that $f^{-1}(y)$ is non-empty and compact. Then, $f^{-1}(y)$ is a finite subset of $M$.

The following results is a consequence of Sard's Theorem for manifolds.

Theorem 26 (Transversality Theorem) Let $M, \Omega$ and $N$ be $C^{r}$ manifolds of dimensions $m, p$ and $n$, respectively. Let $f: M \times \Omega \rightarrow N$ be a $C^{r}$ function. Assume $r>\max \{m-n, 0\}$. If $y \in N$ is a regular value for $f$, then there exists a full measure subset $\Omega^{*}$ of $\Omega$ such that for any $\omega \in \Omega^{*}, y \in N$ is a regular value for $f_{\omega}$, where

$$
f_{\omega}: \xi \in M \rightarrow f_{\omega}(\xi):=f(\xi, \omega) \in N
$$

Definition 27 Let $(X, d)$ and $\left(Y, d^{\prime}\right)$ be two metric spaces. A function $\pi$ : $X \rightarrow Y$ is proper if it is continuous and one among the following conditions holds true.

(1) $\pi$ is closed and $\pi^{-1}(y)$ is compact for each $y \in Y$,

(2) if $K$ is a compact subset of $Y$, then $\pi^{-1}(K)$ is a compact subset of $X$,

(3) if $\left(x^{n}\right)_{n \in \mathbb{N}}$ is a sequence in $X$ such that $\left(\pi\left(x^{n}\right)\right)_{n \in \mathbb{N}}$ converges in $Y$, then $\left(x^{n}\right)_{n \in \mathbb{N}}$ has a converging subsequence in $X$. 
The above conditions are equivalent.

Theorem 28 (Implicit Function Theorem) Let $M, N$ be $C^{r}$ manifolds of the same dimension. Assume $r \geq 1$. Let $(X, \tau)$ be a topological space, and $f$ : $M \times X \rightarrow N$ be a continuous function such that $D_{\xi} f(\xi, x)$ exists and it is continuous on $M \times X$. If $f(\xi, x)=0$ and $D_{\xi} f(\xi, x)$ is onto, then there exist an open neighborhood $I$ of $x$ in $X$, an open neighborhood $U$ of $\xi$ in $M$ and a continuous function $g: I \rightarrow U$ such that $g(x)=\xi$ and $f\left(\xi^{\prime}, x^{\prime}\right)=0$ holds for $\left(\xi^{\prime}, x^{\prime}\right) \in U \times I$ if and only if $\xi^{\prime}=g\left(x^{\prime}\right)$.

\section{References}

Allen, B., 1981. Utility perturbations and the equilibrium price set. Journal of Mathematical Economics 8, 277-307.

Balasko, Y., 1988. Foundations of the General Equilibrium Theory. Academic Press, Boston.

Balasko, Y., Cass, D., Siconolfi, P., 1990. The structure of financial equilibrium with exogenous yields: the case of restricted participation. Journal of Mathematical Economics 19, 195-216.

Bonnisseau, J.-M., 2003. Regular economies with non-ordered preferences. Journal of Mathematical Economics 39, 153-174.

Carosi, L., Villanacci, A., 2005. Relative wealth dependent restricted participation on financial markets. Report 271, University of Pisa, Italy.

Cass, D., Citanna, A., 1998. Pareto improving financial innovation in incomplete markets. Economic Theory 11, 467-494.

Cass, D., Siconolfi, P., Villanacci, A., 2001. Generic regularity of competitive equilibria with restricted participation. Journal of Mathematical Economics $36,61-76$.

Citanna, A., Kajii, A., Villanacci, A., 1998. Constrained suboptimality in incomplete markets: a general approach and two applications. Economic Theory $11,495-522$.

Citanna, A., Polemarchakis, H. M., Tirelli, M., 2006. The taxation of trades in assets. Journal of Economic Theory 126, 299-313.

Crés, H., 1996. Symmetric smooth consumption externalities. Journal of Economic Theory 69, 334-366.

Debreu, G., 1983. Mathematical economics. Cambridge University Press.

Florenzano, M., 2003. General equilibrium analysis, existence and optimality properties of equilibria. Kluwer Academic Publishers.

Geanakoplos, J. D., Polemarchakis, H. M., 1986. Existence, regularity and constrained suboptimality of competitive allocations when the asset market is incomplete. In: Starrett, D., Heller, W. P., Starr, R. M. (eds.), Uncertainty, Information and Communication: Essays in Honor of K. J. Arrow, vol. III, 65-96. Cambridge University Press. 
Geanakoplos, J. D., Polemarchakis, H. M., 2008. Pareto improving taxes. Journal of Mathematical Economics 44, 682-696.

Guillemin, V., Pollack, A., 1974. Differential topology. Prentice-Hall, Englewood Cliffs, NJ.

Hammond, P., 1998. The Efficiency Theorems and Market Failure. In A.P. Kirman (ed.) Elements of General Equilibrium Analysis, ch. 6, 211-260. Oxford: Basil Blackwell.

Heidhues, P., Riedel, F., 2007. Do social preferences matter in competitive markets? Working paper 392, Institute of Mathematical Economics, University of Bielefeld.

Herings, P. J.-J., Polemarchakis, H., 2005. Pareto improving price regulation when the asset market is incomplete. Economic Theory 25, 135-154.

Hirsch, M. W., 1976. Differential topology. Springer-Verlag, New York.

Kung, F.-C., 2008. Voluntary contributions to multiple public goods in a production economy with widespread externalities. Journal of Mathematical Economics 44, 1364-1378.

Laffont, J.-J., 1976. Decentralization with externalities. European Economic Review 7, 359-375.

Laffont, J.-J., 1977. Effets externes et théorie é conomique. Monographies du Séminaire d'Econométrie. Editions du CNRS, Paris, France.

Laffont, J.-J., 1988. Fundamentals of Public Economics. The MIT Press Cambridge.

Laffont, J.-J., Laroque, G., 1972. Effets externes et théorie de l'équilibre général, Cahiers du Séminaire d'Econom étrie 14. CNRS, Paris, France.

Mas-Colell, A., 1985. The theory of general economic equilibrium. A differentiable approach. Cambridge University Press.

del Mercato, E. L., 2006. Existence of competitive equilibria with externalities: A differential viewpoint. Journal of Mathematical Economics 42, 525-543.

Polemarchakis, H., Siconolfi, P., 1997. Generic existence of competitive equilibria with restricted participation. Journal of Mathematical Economics 28, 289-311.

Siconolfi, P., 1986. Equilibrium with restricted participation on incomplete financial markets. CARESS Working Paper 86-21, University of Pennsylvania.

Siconolfi, P., 1988. Equilibrium with asymmetric constraints on portfolio holdings and incomplete financial markets. In: Non-Linear Dynamics in Economics and Social Sciences - Proceeding of the Meeting held in Certosa di Pontignano, January 7-9, 1988, Società Pitagora, Italy.

Smale, S., 1974. Global analysis and economies IV; Finiteness and stability of equilibria with general consumption sets and production. Journal of Mathematical Economics 1, 119-127.

Smale, S., 1981. Global analysis and economics. In: Arrow, K., Intriligator, M. (Eds.), Handbook of Mathematical Economics, vol. I. North- Holland, Amsterdam, Chapter 8, 331-370.

Villanacci, A., Carosi, L., Benevieri, P., Battinelli, A., 2002. Differential topol- 
ogy and general equilibrium with complete and incomplete markets. Kluwer Academic Publishers. 